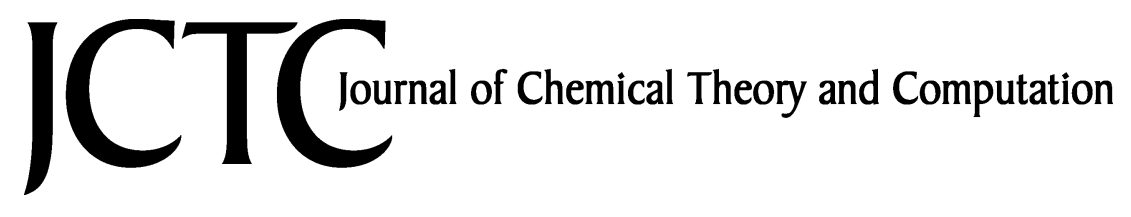

\title{
Validation of Molecular Dynamics Simulations of Biomolecules Using NMR Spin Relaxation as Benchmarks: Application to the AMBER99SB Force Field
}

\author{
Scott A. Showalter and Rafael Brüschweiler* \\ Department of Chemistry and Biochemistry and National High Magnetic Field \\ Laboratory, Florida State University, Tallahassee, Florida 32306
}

Received January 5, 2007

\begin{abstract}
Biological function of biomolecules is accompanied by a wide range of motional behavior. Accurate modeling of dynamics by molecular dynamics (MD) computer simulations is therefore a useful approach toward the understanding of biomolecular function. NMR spin relaxation measurements provide rigorous benchmarks for assessing important aspects of MD simulations, such as the amount and time scales of conformational space sampling, which are intimately related to the underlying molecular mechanics force field. Until recently, most simulations produced trajectories that exhibited too much dynamics particularly in flexible loop regions. Recent modifications made to the backbone $\varphi$ and $\psi$ torsion angle potentials of the AMBER and CHARMM force fields indicate that these changes produce more realistic molecular dynamics behavior. To assess the consequences of these changes, we performed a series of 5-20 ns molecular dynamics trajectories of human ubiquitin using the AMBER99 and AMBER99SB force fields for different conditions and water models and compare the results with NMR experimental backbone $\mathrm{N}-\mathrm{H} S^{2}$ order parameters. A quantitative analysis of the trajectories shows significantly improved agreement with experimental NMR data for the AMBER99SB force field as compared to AMBER99. Because NMR spin relaxation data ( $T_{1}$, $T_{2}$, NOE) reflect the combined effects of spatial and temporal fluctuations of bond vectors, it is found that comparison of experimental and back-calculated NMR spin-relaxation data provides a more objective way of assessing the quality of the trajectory than order parameters alone. Analysis of a key mobile $\beta$-hairpin in ubiquitin demonstrates that the dynamics of mobile sites are not only reduced by the modified force field, but the extent of motional correlations between amino acids is also markedly diminished.
\end{abstract}

\section{Introduction}

NMR spin relaxation spectroscopy and molecular dynamics (MD) computer simulations are both widely used tools to study the dynamics of biomolecules. Although both methods probe motions on the picosecond to nanosecond range, they are highly complementary in many aspects. Spin relaxation is sensitive to reorientational dynamics of the lattice functions of spin-relaxation active interactions, such as $\mathrm{N}-\mathrm{H}$ bond vectors belonging to ${ }^{15} \mathrm{~N}-{ }^{1} \mathrm{H}$ magnetic dipole-dipole inter-

* Corresponding author tel.: 850-644-1768; fax: 850-644-8281; e-mail: bruschweiler@magnet.fsu.edu. actions, while MD simulations provide information on the motions of all atoms of a biomolecule. ${ }^{1-4}$ Due to the strong dependence of the MD simulation on the applied force field and the computational protocol, ${ }^{5}$ validation of simulations against experimental data is critically important. NMR spin relaxation data reflect motional amplitudes and time scales of both overall tumbling and internal motions for many sites throughout a protein and are therefore excellently suited for this task.

Comparisons of MD simulations ${ }^{6-16}$ with NMR relaxation data typically focus on the generalized $\mathrm{N}-\mathrm{H} S^{2}$ order 
parameter by Lipari and Szabo. ${ }^{17,18} S^{2}$ is a measure of the spatial restriction of the $\mathrm{N}-\mathrm{H}$ vector in a molecule-fixed frame that takes values between 0 (large amounts of motion) and 1 (no motion) and which can be extracted from longitudinal $T_{1}$, transverse $T_{2}$, and heteronuclear Overhauser effect (NOE) data. Due to the underlying spin physics, the experimental $S^{2}$ order parameters only report on motions that occur on time scales comparable to or faster than the overall tumbling correlation time $\tau_{\mathrm{c}}$ of the biomolecule, which is typically in the low nanosecond range. Such comparisons between MD simulations and NMR relaxation have been hampered in two important ways. First, due to the limited available computer power, MD trajectories have typically been much shorter than the overall tumbling correlation time and therefore did only reflect a subset of motions observed in NMR relaxation experiments. Therefore, the computed order parameters were on average higher than the ones of longer simulations using the same force field. Second, computed order parameters belonging to loop regions were often found to be substantially lower than their experimental counterparts, raising questions about the adequacy of the applied computational protocols or force fields. It is important to note that because these two kinds of errors have the tendency to cancel each other at least partially, agreement between calculated and experimental $S^{2}$ values could be accidental without validating the quality of the force field itself.

At the same time, the development of simplified contact models led to the remarkably accurate prediction of $S^{2}$ profiles from average 3D structures relying on very few adjustable parameters. ${ }^{19-21}$ This shows that the problem is generally not rooted with local features of the structural model but rather with the MD force field itself.

The situation has started to change recently due to the availability of increased computer power and adjustments made to the force fields. A correction to the CHARMM C22 force field, called CMAP, was introduced, which modifies the potential of the backbone torsion angles $\varphi$ and $\psi \cdot{ }^{22}$ The use of this corrected force field was found to improve the overall agreement between MD-derived and experimental NMR order parameters of hen lysozyme, although some discrepancies remain. ${ }^{23}$ A similar modification was introduced into the AMBER99 force field with the result that an implicit solvent trajectory of HIV-1 protease yielded dynamics in good agreement with experimental data for that system. ${ }^{24}$ Improvements provided by the AMBER99SB force field at reproducing experimental order parameters have been further demonstrated for hen lysozyme and human ubiquitin. ${ }^{25}$

As MD simulations now routinely exceed in length the overall tumbling correlation times $\tau_{\mathrm{c}}$, order parameters computed over the whole trajectory include motions that are too slow to affect in significant ways $T_{1}, T_{2}$, and $\mathrm{NOE}$ relaxation parameters. Because these motions would not be reflected in the experimental $S^{2}$ values, their effect on calculated $S^{2}$ values introduces a bias and makes the comparison of experimental and simulation results at the order parameter level not straightforward. In fact, recent residual dipolar coupling measurements suggest that ad- ditional motions are present on nanosecond to millisecond time scales that are not reflected in relaxation-derived $S^{2}$ values. Therefore, as MD trajectories are increasing in length, a further drop in $S^{2}$ values, at least for some protein systems, is expected.

An alternative approach for assessing the quality of a protein MD trajectory directly compares experimental and predicted $T_{1}, T_{2}$, and NOE relaxation parameters by leaving out the $S^{2}$ order parameter comparison as an intermediate step. ${ }^{16,26-28}$ This approach requires both a proper description of internal motions as well as an accurate modeling of overall tumbling. A related approach directly compares spectral density components $J(\omega),{ }^{16}$ whereby the extraction of spectral densities from the experiment requires additional experiments or makes additional assumptions. ${ }^{29}$

Here, we report the results of molecular dynamics trajectories of the well-characterized protein ubiquitin computed in the AMBER software package, ${ }^{30}$ using both the AMBER99 $^{31}$ and the modified AMBER99SB ${ }^{24,25}$ force fields. Several sets of experimental NMR spin relaxation data have been published for ubiquitin, ${ }^{32-36}$ making it a valuable model system for assaying the quantitative accuracy of the MDderived NMR relaxation parameters. Ubiquitin has also been successfully used as a model system for the MD investigation of NMR relaxation active motion in the past, yielding fundamental insight into the nature of internal protein dynamics. ${ }^{34,37,38}$ More recently, a $0.2 \mu$ s ubiquitin MD trajectory calculated using the GROMOS96 $43 \mathrm{a} 1$ force field $^{39}$ was reported by Nederveen and Bonvin (referred to as the "NB trajectory" from here on) from which both order parameters and spin relaxation parameters were computed. ${ }^{28}$ The results of the current work indicate that the modified AMBER99SB force field performs qualitatively better than previous force fields at reproducing experimental order parameters, and it yields NMR spin relaxation parameters in near-quantitative agreement with experimental values.

\section{Materials and Methods}

2.1. Molecular Dynamics Simulations. Explicit solvent MD trajectories were run using the AMBER 8.0 software package $^{30}$ with the AMBER99 ${ }^{31}$ and the AMBER99SB ${ }^{24,25}$ force field under particle mesh Ewald periodic boundary conditions. ${ }^{40}$ Two initial configurations were generated in which ubiquitin (PDB code 1UBQ) ${ }^{41}$ was solvated with 6080 water molecules represented with either the SPC or TIP3P mode $l^{42}$ such that no solute atom was within $12 \AA$ of a box edge. The four resulting systems (AMBER99 with SPC, AMBER99 with TIP3P, AMBER99SB with SPC, and AMBER99SB with TIP3P) were energy-minimized and heated to $50 \mathrm{~K}$ through $10 \mathrm{ps}$ of canonical (NVT) MD, with a 2 fs time step and SHAKE constraints on bonds involving hydrogen atoms ${ }^{43}$ followed by heating to 150 and $300 \mathrm{~K}$ in successive 20 ps steps under the same conditions. Next, the systems were allowed to equilibrate in a $50 \mathrm{ps}$ isothermalisobaric (NPT) simulation in order to equilibrate the solvent density. After this initial equilibration period, an additional $5.0 \mathrm{~ns}$ of production MD was performed on each of the four systems under NPT conditions, and separately, $5.0 \mathrm{~ns}$ of production MD was performed under NVT conditions. The 
result was a set of eight $5.1 \mathrm{~ns}$ trajectories, spanning all combinations of the two force fields, two water models, and two production run conditions. Snapshots of each trajectory were stored to disk every $1.0 \mathrm{ps}$ during both the equilibration and production periods. Finally, the AMBER99 and AMBER99SB trajectories computed under NPT conditions with the SPC water model were extended for a further 15.0 ns yielding final trajectories $20.1 \mathrm{~ns}$ in length. Superposition of the snapshots to remove the effects of overall translation and rotation was performed within the AMBER ptraj module by superimposing the backbone heavy atoms of residues $1-72$, using the snapshot at the midpoint of the production run $(2.5$ or $10.0 \mathrm{~ns})$ as the reference structure.

2.2. Correlations Functions and Order Parameters. Bloch-Wangsness-Redfield spin relaxation theory ${ }^{44-47}$ expresses spin-relaxation rates induced by reorientational motion in terms of the spectral density functions:

$$
J(\omega)=2 \int_{0}^{\infty} C(t) \cos (\omega t) \mathrm{d} t
$$

where $C(t)$ is the time correlation function of the lattice portion of the spin interaction causing relaxation. For the relaxation of ${ }^{15} \mathrm{~N}$ spins in proteins, the relevant interactions are the magnetic dipole-dipole interaction with the covalently bonded proton spin and the ${ }^{15} \mathrm{~N}$ chemical shielding anisotropy. The principal axes of the tensors describing these two interactions are assumed to be collinear and parallel with the ${ }^{15} \mathrm{~N}-{ }^{1} \mathrm{H}$ bond vector. For a protein in isotropic solution, the reorientational correlation function in eq 1 takes the form

$$
C(t)=\left\langle P_{2}\left[\mathbf{e}_{\mathrm{LF}}(\tau) \mathbf{e}_{\mathrm{LF}}(\tau+t)\right]\right\rangle
$$

where $P_{2}$ is the second Legendre polynomial $\left[P_{2}=\right.$ $\left.1 / 2\left(3 x^{2}-1\right)\right], \mathbf{e}_{\mathrm{LF}}$ is a unit vector collinear with the bond vector defined relative to the laboratory frame (LF), and the angular brackets indicate averaging over time $\tau$.

The "model-free" formalism developed by Lipari and Szabo for parametrization of the functions described in eqs 1 and 2 requires that any internal motions of the interaction vector be separable from global tumbling of the molecule. ${ }^{17,18}$ For isotropic overall tumbling, eq 2 may then be factored as

$$
C(t)=C_{\mathrm{O}}(t) C_{\mathrm{I}}(t)
$$

where $C_{\mathrm{O}}(t)$ and $C_{\mathrm{I}}(t)$ describe global and internal reorientation, respectively, where the correlation function $C_{\mathrm{O}}(t)$ is

$$
C_{\mathrm{O}}(t)=\mathrm{e}^{-t / \tau_{c}}
$$

where $\tau_{\mathrm{c}}$ is the rank 2 correlation time for global tumbling. The internal correlation function is approximated by ${ }^{17,18}$

$$
C_{\mathrm{I}}(t)=\left\langle P_{2}[\mathbf{e}(\tau) \mathbf{e}(\tau+t)]\right\rangle \cong S^{2}+\left(1-S^{2}\right) \mathrm{e}^{-t / \tau_{e}}
$$

where the unit vector $\mathbf{e}$ is defined relative to a fixed internal frame. $C_{\mathrm{I}}(t)$, which is normalized $\left[C_{\mathrm{I}}(0)=1\right]$, decays with an effective correlation time $\left(\tau_{\mathrm{e}}\right)$ to a plateau value $S^{2}$, the square of the generalized order parameter. The angular brackets indicate averaging over time $\tau$. $S^{2}$ represents the spatial restriction of the motion of the interaction vector and is determined by ${ }^{17,18,48}$

$$
C_{\mathrm{I}}(\infty)=S^{2}=1-\frac{4 \pi}{5} \sum_{m=-2}^{2} \sigma_{Y_{2 m}}^{2}
$$

where $\sigma_{Y_{2 m}}{ }^{2}$ are the variances of the normalized spherical harmonics of rank $2, Y_{2 m}[\theta(t), \varphi(t)]$, over the trajectory and $\theta(t)$ and $\varphi(t)$ are the polar angles representing the interaction vector relative to a fixed internal frame. Equation 5 is valid for monoexponentially decaying correlation functions with a correlation time faster than the overall tumbling correlation time $\tau_{\mathrm{c}}$ or for multiexponential correlation functions with all correlation times much shorter than $\tau_{\mathrm{c}}$.

Recently, a general method for calculating NMR spin relaxation parameters from MD trajectories, known as isotropic reorientational eigenmode dynamics (iRED), was introduced. ${ }^{38}$ In this method, the snapshots generated from the MD trajectory are treated analytically to yield an isotropic ensemble from which a covariance matrix $\mathbf{M}$ is computed with elements

$$
M_{i j}=\frac{1}{2}\left\langle 3\left(\mathbf{e}_{\mathrm{LF}, i} \mathbf{e}_{\mathrm{LF}, j}\right)^{2}-1\right\rangle
$$

where $\mathbf{e}_{\mathrm{LF}, i}$ and $\mathbf{e}_{\mathrm{LF}, j}$ are the normalized bond vectors, taken from the same snapshot, and the brackets indicate averaging over all snapshots. Because eq 7 contains only inner products, $M_{i j}$ is rotationally invariant; that is, it is valid in an arbitrary frame that makes no assumptions about separability between internal and overall tumbling motions. $S^{2}$ values can be computed from $\mathbf{M}$ by solving the eigenvalue problem $\mathbf{M} \mid m>$ $=\lambda_{m} \mid m>(m=1, \ldots, N$, where $N=$ number of interaction vectors $\mathbf{e}_{m}$ ) and using ${ }^{38}$

$$
\left.S^{2}=1-\sum_{m=1}^{N-5} \lambda_{m}|| m\right\rangle\left._{j}\right|^{2}
$$

where the sum extends over the N-5 eigenvectors $|m\rangle$ corresponding to internal modes with eigenvalues $\lambda_{m}$ (i.e., all eigenvectors except those with the five largest eigenvalues). Equation 8 provides a computationally efficient way to determine order parameters without requiring the explicit removal of overall tumbling from the trajectory.

2.3. Spectral Density Functions and Spin Relaxation. To obtain an accurate estimate for $\tau_{\mathrm{c}}$ from a MD trajectory, the trajectory's length must exceed $\tau_{\mathrm{c}}$ by at least $1-2$ orders of magnitude, which poses a challenge even for modern computers. Moreover, MD simulations tend to significantly underestimate $\tau_{\mathrm{c}}$. Therefore, when constructing the overall correlation function $C(t)$ from the trajectory, it is often advantageous to first compute $C_{\mathrm{I}}(t)$ from the trajectory and model $C_{\mathrm{O}}(t)$ with a $\tau_{\mathrm{c}}$ determined experimentally. $C_{\mathrm{I}}(t)$ is computed using the left part of eq 5 after first removing overall tumbling by superimposing each snapshot with respect to a reference snapshot.

Once $C_{\mathrm{I}}(t)$ is computed, it can be converted by Fourier transform (eq 1) into the spectral density function needed to calculate NMR spin relaxation parameters. For this purpose, it is convenient to fit the numerical correlation function to a set of exponentials, so that the spectral density function can 
be expressed analytically as a sum of Lorentzians. ${ }^{27}$ The internal correlation functions are computed for the interval $t=0, \ldots, 0.3 T_{\mathrm{MD}}$, where $T_{\mathrm{MD}}$ is the total length of the molecular dynamics trajectory. Each correlation function is then fit to a multiexponential function: ${ }^{27}$

$$
C_{\mathrm{I}}(t)=A_{0}+\sum_{k=1}^{5} A_{k} \mathrm{e}^{-t / \tau_{k}}
$$

subject to the normalization condition that $\sum_{j} A_{j}=1.0(j=$ $0, \ldots, 5), A_{k} \geq 0$, and $0 \leq \tau_{k}$ for all $k$. Because it captures the long correlation time behavior of $C_{\mathrm{I}}(t), A_{0}$ is analogous to $S^{2}$ from the Lipari-Szabo model (eq 5).

The best-fit parameters generated from fitting the MD correlation functions to eq 9 are used in combination with the experimental value of $\tau_{\mathrm{c}}$ to calculate the spectral density function:

$$
J(\omega)=\frac{A_{0} 2 \tau_{\mathrm{c}}}{1+\left(\omega \tau_{\mathrm{c}}\right)^{2}}+\sum_{k=1}^{5} \frac{A_{k} 2 \tau^{\prime}}{1+\left(\omega \tau^{\prime}\right)^{2}}
$$

where $\tau^{\prime}=\tau_{\mathrm{c}} \tau_{k} /\left(\tau_{\mathrm{c}}+\tau_{k}\right)$. The spectral density functions are then used to compute the NMR observables ${ }^{15} \mathrm{~N} \mathrm{~T}_{1},{ }^{15} \mathrm{~N} \mathrm{~T}_{2}$, and the ${ }^{1} \mathrm{H}-{ }^{15} \mathrm{~N}$ NOE using the following well-known equations: ${ }^{49}$

$$
\begin{gathered}
\frac{1}{T_{1}}=R_{1}=d_{o o}\left[3 J\left(\omega_{\mathrm{N}}\right)+J\left(\omega_{\mathrm{H}-\mathrm{N}}\right)+6 J\left(\omega_{\mathrm{N}+\mathrm{H}}\right)\right]+ \\
c_{o o} \omega_{\mathrm{N}}^{2} J\left(\omega_{\mathrm{N}}\right) \\
\frac{1}{T_{2}}=R_{2}=\frac{1}{2} d_{o o}\left[4 J(0)+3 J\left(\omega_{\mathrm{N}}\right)+J\left(\omega_{\mathrm{H}-\mathrm{N}}\right)+6 J\left(\omega_{\mathrm{H}}\right)+\right. \\
\left.6 J\left(\omega_{\mathrm{N}+\mathrm{H}}\right)\right]+\frac{1}{6} c_{o o} \omega_{\mathrm{N}}^{2}\left[4 J(0)+3 J\left(\omega_{\mathrm{N}}\right)\right] \\
\mathrm{NOE}=1+\frac{\gamma_{\mathrm{H}}}{\gamma_{\mathrm{N}}} d_{o o} T_{1}\left[6 J\left(\omega_{\mathrm{H}+\mathrm{N}}\right)-J\left(\omega_{\mathrm{H}-\mathrm{N}}\right)\right]
\end{gathered}
$$

where $d_{o o}=(1 / 20)\left(\mu_{o} / 4 \pi\right)^{2}(h / 2 \pi)^{2} \gamma_{\mathrm{N}^{2}} \gamma_{\mathrm{H}^{2}}<r_{\mathrm{NH}}{ }^{-3}>2, c_{o o}=$ $(1 / 15) \Delta \sigma^{2}, \mu_{0}$ is the vacuum permeability, $h$ is Planck's constant, $\gamma_{\mathrm{N}}$ and $\gamma_{\mathrm{H}}$ are the gyromagnetic ratios of ${ }^{15} \mathrm{~N}$ and ${ }^{1} \mathrm{H}$, respectively, $r_{\mathrm{NH}}=1.02 \AA$ is the $\mathrm{N}-\mathrm{H}$ bond length, and $\Delta \sigma=-160 \mathrm{ppm}$ is the chemical shift anisotropy of ${ }^{15} \mathrm{~N}$ in an amide group. $\omega_{\mathrm{N}}, \omega_{\mathrm{H}}, \omega_{\mathrm{N}+\mathrm{H}}$, and $\omega_{\mathrm{H}-\mathrm{N}}$ are the Larmor frequencies as well as their sum and difference in radians per second of ${ }^{15} \mathrm{~N}$ and ${ }^{1} \mathrm{H}$ spins, respectively.

The quality of the agreement between experimental and calculated values of $T_{1}, T_{2}$, and NOE was assessed in a way that is independent of their relative magnitudes. For this purpose, we define a parameter $Q_{\mathrm{P}}$ in analogy to the $Q$ value used for residual dipolar couplings: ${ }^{50}$

$$
Q_{\mathrm{P}}=\frac{\left[\frac{1}{N} \sum_{i=1}^{N}\left(P_{i}^{\mathrm{obs}}-P_{i}^{\mathrm{pred}}\right)^{2}\right]^{1 / 2}}{\left[\frac{1}{N} \sum_{i=1}^{N}\left(P_{i}^{\mathrm{obs}}\right)^{2}\right]^{1 / 2}}
$$

where $P^{\mathrm{obs}}$ and $P^{\text {pred }}$ are the experimentally and MD-derived values, respectively, of either the parameter set $P_{T}=T_{1}, T_{2}$, and NOE or set $P_{R}=R_{1}, R_{2}$, and NOE, and the sum runs over the residues for which both experimental and MD data are available. For $P_{T}$, mobile residues with large $T_{1}$ and $T_{2}$ values are emphasized, whereas for $P_{R}$, the influence of these residues on $Q$ is diminished.

2.4. Torsion Angle Correlations. As the AMBER 99 and AMBER99SB force fields differ in their $\varphi$ and $\psi$ torsion angle potentials,${ }^{25}$ it is important to understand what (if any) influence the reparametrization has on the extent of correlated motions among these torsion angles. A complex covariance matrix $\mathbf{C}$ can be defined with elements ${ }^{51}$

$$
\begin{aligned}
C_{k, l}=\operatorname{cov}\left(\cos \theta_{k}, \cos \theta_{l}\right)+\operatorname{cov}\left(\sin \theta_{k}, \sin \theta_{l}\right)+ \\
i \operatorname{cov}\left(\cos \theta_{k}, \sin \theta_{l}\right)-i \operatorname{cov}\left(\sin \theta_{k}, \cos \theta_{l}\right)
\end{aligned}
$$

where $\operatorname{cov}(f, g)=\left\langle f^{*} g\right\rangle-\left\langle f^{*}>\langle g\rangle\right.$, where $f$ and $g$ are functions of the torsion angles $\theta_{k, l}$ ( $\varphi$ and $\psi$ for each residue). In contrast to $\operatorname{cov}\left(\theta_{k}, \theta_{l}\right)$, eq 15 does not depend on the choice of the origin of the torsion angles $\theta_{k}$ and $\theta_{l}$.

\section{Results and Discussion}

\subsection{Overall Effects of the AMBER99 and AMBER99SB}

Force Fields. Analysis of the ubiquitin molecular dynamics trajectories computed with both the AMBER99 ${ }^{31}$ and the AMBER99SB ${ }^{24,25}$ force fields indicates that all simulations are stable over the course of the production runs. The backbone $\mathrm{C}^{\alpha}$ atom root-mean-square difference (RMSD) of each snapshot with respect to the crystal structure (excluding the mobile C-terminal tail residues 73-76) shows that, after an initial equilibration period, each of the trajectories represents an ensemble with similar RMSD values. The RMSD traces for the AMBER99 and AMBER99SB trajectories with the SPC water model under NPT conditions, which will be discussed in greater detail below, are shown in Figure 1A. The $\mathrm{C}^{\alpha}$ RMSDs of the individual residues, which provide site-specific information regarding the extent of motion sampled throughout the protein backbone, shows clear differences between these two trajectories (Figure 1B): the extent of fluctuations in several of the loops is larger with AMBER99 than with AMBER99SB. The rigidification of the backbone torsion angles $\varphi$ and $\psi$ in AMBER99SB ${ }^{25}$ is also reflected in the average angular $\operatorname{RMSDs}$ of $\varphi$ and $\psi$ in the hairpin loop (residues 7-10), which decreases from $20.1^{\circ}$ to $16.3^{\circ}$ for AMBER99SB in an NPT simulation with SPC water, while the angular RMSD values for the secondary structural elements, which are intrinsically more rigid, remain unchanged at about $7-12^{\circ}$ in both trajectories.

3.2. Comparison of Calculated Order Parameters with Experiment. Order parameters computed from MD trajectories are commonly used as a metric for comparing dynamics found in experiments and simulations. Such comparison focuses on the spatial aspects of motion but does not provide direct information on agreement of the corresponding time scales. Besides the length of the trajectory, several factors in the simulation may influence the calculated order parameters including the force field, the choice of water model, and the choice to run NVT- or NPT-type dynamics. The impact of these factors was assessed by calculating a total of eight trajectories combining the AMBER99 or 
A

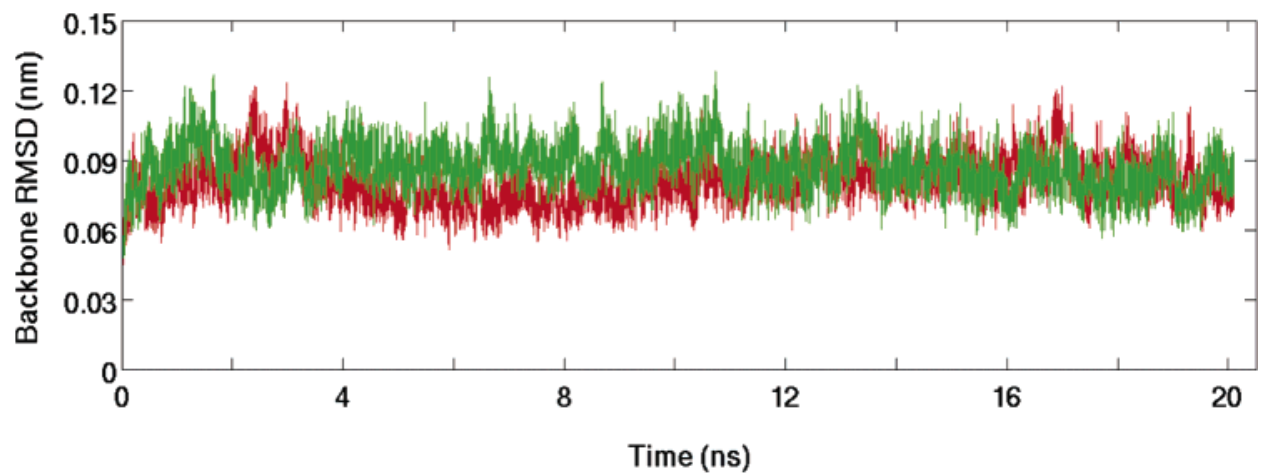

B

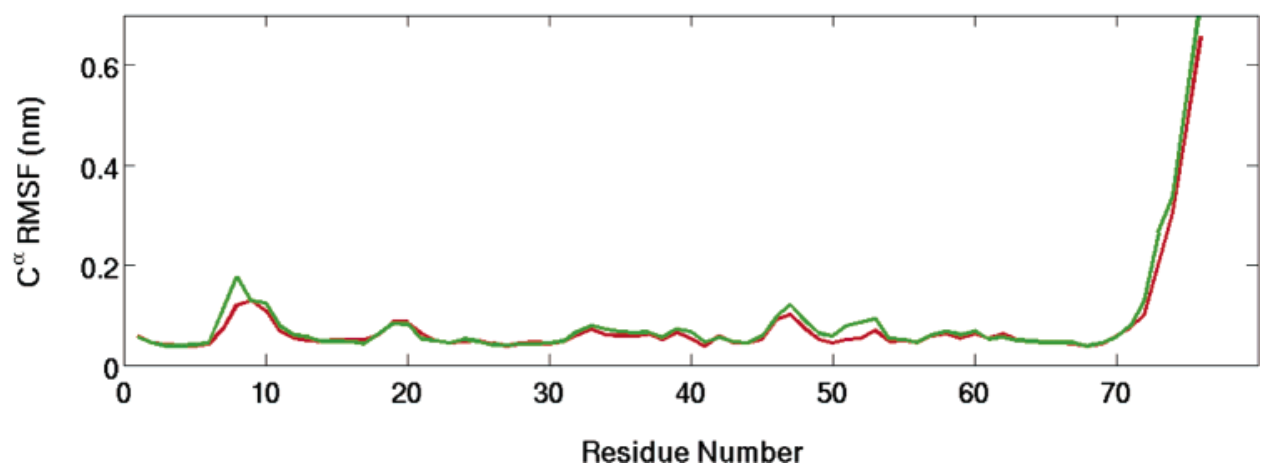

Figure 1. Backbone fluctuations over the 20 ns trajectories computed with AMBER99 (green) and AMBER99SB (red) under NPT conditions with SPC water. The backbone RMSD for all residues (A) is comparable between the two trajectories. The AMBER99 trajectory experiences larger fluctuations for many of the residues in mobile regions (B), although the backbone RMSD is similar for many residues of the most rigid regions of ubiquitin.

Table 1. Statistical Comparison of Order Parameters Calculated from Each of the 5 ns MD Trajectories with the Experimental Data Set ${ }^{34}$

\begin{tabular}{lcccc}
\hline \multicolumn{1}{c}{ data set } & $R_{\mathrm{p}}{ }^{a}$ & $R_{\mathrm{s}}{ }^{b}$ & $\mathrm{RMSD}^{c}$ & $<S_{\mathrm{MD}^{2} / S_{\mathrm{NMR}^{2}}>d}$ \\
\hline AMBER99, SPC, NPT & 0.817 & 0.784 & 0.069 & $0.99 \pm 0.11$ \\
AMBER 99, SPC, NVT & 0.776 & 0.785 & 0.078 & $0.99 \pm 0.12$ \\
AMBER 99, TIP3P, NPT & 0.901 & 0.763 & 0.086 & $0.95 \pm 0.15$ \\
AMBER 99, TIP3P, NVT & 0.901 & 0.765 & 0.076 & $0.96 \pm 0.12$ \\
AMBER 99SB, SPC, NPT & 0.921 & 0.794 & 0.042 & $1.02 \pm 0.05$ \\
AMBER 99SB, SPC, NVT & 0.919 & 0.766 & 0.048 & $1.02 \pm 0.07$ \\
AMBER 99SB, TIP3P, NPT & 0.946 & 0.811 & 0.036 & $1.02 \pm 0.04$ \\
AMBER 99SB, TIP3P NVT & 0.964 & 0.826 & 0.044 & $1.01 \pm 0.10$ \\
experimental set & 0.981 & 0.902 & 0.026 & $0.99 \pm 0.05$ \\
\hline
\end{tabular}

a Pearson's correlation coefficient between the simulation and the experimental set. ${ }^{34}{ }^{b}$ Spearman's rank correlation coefficient between the simulation and the experimental set. ${ }^{34}{ }^{c}$ Root-mean-square deviation between the simulation and the experimental set. ${ }^{34}{ }^{d} \mathrm{Av}$ erage is taken over all residues for which there are both MD and experimental data.

AMBER99SB force fields, with either the SPC or TIP3P water models, running either under NVT or NPT conditions. All eight trajectories combining these three parameter pairs were run for $5 \mathrm{~ns}$ of production dynamics and then analyzed to yield $S^{2}$ for each backbone $\mathrm{N}-\mathrm{H}$ bond vector. The MDderived order parameters are compared with the 61 experimentally determined values from Lienin et al. ${ }^{34}$ used here as a reference. Statistics of the comparison are summarized in Table 1. In general, AMBER99SB trajectories have higher correlation coefficients, a lower RMSD, and a lower standard deviation in the $S_{\mathrm{MD}}{ }^{2} / S_{\mathrm{NMR}}{ }^{2}$ ratio than the corresponding AMBER99 trajectories, demonstrating that the AMBER99SB force field does a better job of reproducing experimental order parameters. Although the statistics also indicate slightly better results for NPT conditions, the difference between NPT and NVT conditions is minimal compared to the difference caused by changing the force field. Comparing the results for the SPC and TIP3P water models suggests that the choice of water model has some, albeit small, effect on calculated order parameters.

While the agreement between the predicted $S^{2}$ values using AMBER99SB and the experimental results of Lienin et al. is overall good, ${ }^{34}$ it is important to know if further improvements can realistically be expected. Tjandra et al..$^{33}$ have reported ubiquitin order parameters under conditions very similar to those of the Lienin et al. ${ }^{34}$ data set. The bottom row of Table 1 shows that these two experimental data sets are well-comparable, reflecting good reproducibility and high quality, and they provide information about inherent uncertainties in experimentally derived order parameters. The correlation between the two experimental data sets is clearly higher than the correlation between the experimental data and the best MD-derived data set, which helps establish an upper limit for the agreement between simulations and experiments that can be expected under optimal circumstances.

The average $S_{\mathrm{MD}}{ }^{2} / S_{\mathrm{NMR}^{2}}{ }^{2}$ ratio (see the final column of Table 1) suggests that the AMBER99 trajectories slightly overestimate the dynamics of ubiquitin, while the AMBER99SB trajectories slightly underestimate them (although in both cases the difference from a 1:1 ratio is less than the standard deviation over the data set). Ratios less than one for the AMBER99 simulations are consistent with a body of literature reporting that MD trajectories based on 
Table 2. Order Parameter Statistics as a Function of Trajectory Duration with Respect to the Experimental Set ${ }^{34}$

\begin{tabular}{lcccc}
\hline \multicolumn{1}{c}{ data set } & $R_{\mathrm{p}}{ }^{a}$ & $R_{\mathrm{s}}{ }^{b}$ & $\mathrm{RMSD}^{c}$ & $<\mathrm{SMD}^{2} / S_{\mathrm{NMR}^{2}>d}$ \\
\hline AMBER 99, 500 ps & 0.775 & 0.722 & 0.066 & $1.05 \pm 0.16$ \\
AMBER 99, 1 ns & 0.791 & 0.741 & 0.062 & $1.04 \pm 0.14$ \\
AMBER 99, first 5 ns & 0.817 & 0.784 & 0.069 & $0.99 \pm 0.11$ \\
AMBER 99, second 5 ns & 0.907 & 0.785 & 0.058 & $0.82 \pm 0.11$ \\
AMBER 99, third 5 ns & 0.730 & 0.760 & 0.071 & $1.01 \pm 0.14$ \\
AMBER 99, fourth 5 ns & 0.709 & 0.790 & 0.076 & $1.01 \pm 0.16$ \\
AMBER 99, 10 ns & 0.865 & 0.810 & 0.082 & $0.97 \pm 0.11$ \\
AMBER 99, 20 ns & 0.853 & 0.795 & 0.084 & $0.97 \pm 0.12$ \\
AMBER 99SB, 500 ps & 0.916 & 0.733 & 0.051 & $1.04 \pm 0.06$ \\
AMBER 99SB, 1 ns & 0.901 & 0.745 & 0.052 & $1.04 \pm 0.07$ \\
AMBER 99SB, 5 ns & 0.921 & 0.794 & 0.042 & $1.02 \pm 0.05$ \\
AMBER 99SB, second 5 ns & 0.942 & 0.801 & 0.042 & $1.03 \pm 0.11$ \\
AMBER 99SB, third 5 ns & 0.882 & 0.743 & 0.052 & $1.04 \pm 0.07$ \\
AMBER 99SB, fourth 5 ns & 0.808 & 0.760 & 0.060 & $1.04 \pm 0.14$ \\
AMBER 99SB, first 10 ns & 0.938 & 0.807 & 0.039 & $1.02 \pm 0.05$ \\
AMBER 99SB, 20 ns & 0.944 & 0.807 & 0.040 & $1.01 \pm 0.07$ \\
\hline
\end{tabular}

a Pearson's correlation coefficient between the simulation and the experimental set. ${ }^{34}{ }^{b}$ Spearman's rank correlation coefficient between the simulation and the experimental set. ${ }^{34}{ }^{c}$ Root-mean-square deviation between the simulation and the experimental set. ${ }^{34}{ }^{d} \mathrm{Av}$ erage is taken over all residues for which there are both MD and experimental data.

this and similar force fields often underestimate $S^{2}$ in loop regions. ${ }^{1,10,14-16,52}$

The trajectories using the two force fields exhibit differences in their internal motional correlation times, and therefore the total length of the trajectory, $T_{\mathrm{MD}}$, required to produce optimal agreement with experimental $S^{2}$ values is different for the two force fields. To demonstrate this effect, the order parameters were computed from the AMBER99 and AMBER99SB trajectories after $500 \mathrm{ps}$ and 1, 5, 10, and $20 \mathrm{~ns}$ and compared against the experimental values (Table 2). The order parameters from the $20 \mathrm{~ns}$ AMBER 99 and AMBER99SB trajectories are plotted as a function of the residue number, along with the experimental values of Lienin et al. ${ }^{34}$ in Figure 2. For the relatively rigid sites $\left(S^{2} \geq 0.85\right)$, both the AMBER99 and AMBER99SB simulations tend to overestimate $S^{2}$, regardless of the length of the trajectory, which results in $\left\langle S_{\mathrm{MD}}{ }^{2} / S_{\mathrm{NMR}}{ }^{2}>>1.0\right.$ (at least for short trajectories). A previously reported ubiquitin trajectory calculated with the AMBER99SB force field did not display the baseline offset seen here, because the comparison was made against a different experimental data set with slightly higher order parameters, on average, for the rigid sites. ${ }^{25,33}$ Comparison against the Tjandra et al. ${ }^{33}$ experimental values relieves the baseline offset but does not improve the quality of agreement otherwise (data not shown). On average, the GROMOS96 force field reproduces the order parameters of the rigid sites in ubiquitin quite well, ${ }^{28}$ and the CHARMM C22/CMAP force field performs similarly well for the rigid sites of hen lysozyme. ${ }^{23}$

By contrast, mobile regions of ubiquitin, such as the $\beta$-hairpin loop and several of the loops closer to the $\mathrm{C}$ terminus of the protein, turn out to be too floppy in the AMBER99 simulation, whereas they tend to be in good agreement with experimental results in the AMBER99SB simulation (Figure 2). Plots of the AMBER99 order parameters computed after 1 and $20 \mathrm{~ns}$ correlated against the experimental data (Figure $3 \mathrm{~A}$ and $\mathrm{C}$ ) suggest that the $1 \mathrm{~ns}$ order parameters are the best result for the AMBER99 trajectory. This is in contrast to the AMBER99SB trajectory, which appears to improve by most of the applied statistical measures as the trajectory duration increases (Table 2 and Figure 3B,D).

Figure 2 shows that the order parameters for the mobile regions $\left(S^{2}<0.85\right)$ clearly differ between the AMBER99 and AMBER99SB trajectories. While for both force fields these order parameters decrease as the total trajectory time increases (Figure 3), the rate of change is much larger for the AMBER99 force field than for AMBER99SB. Order parameters calculated over $20 \mathrm{~ns}$ show that the dynamics of the mobile regions are too unrestricted in the AMBER99 trajectory (Figures 2 and 3c). A similar behavior has been observed in the NB trajectory. ${ }^{28}$

For AMBER99, it is clear from Table 2 and Figure 3A and $\mathrm{C}$ that the $1 \mathrm{~ns}$ trajectory does a better job of reproducing the dynamics of the mobile regions of ubiquitin than the longer trajectories. The continuous increase of the RMSD as the trajectory duration increases and the accompanying decrease in $\left\langle S_{\mathrm{MD}}{ }^{2} / S_{\mathrm{NMR}}{ }^{2}>\right.$ dominated by the underestimated order parameters of the mobile regions indicates that the

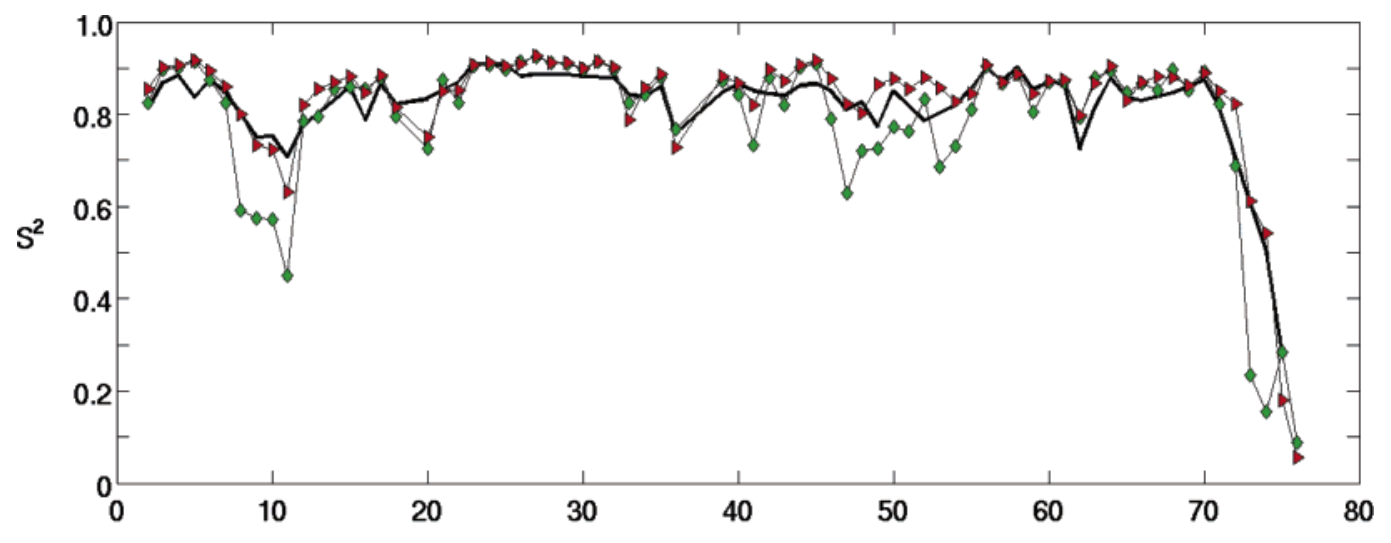

Residue Number

Figure 2. Ubiquitin backbone $\mathrm{N}-\mathrm{H}$ bond order parameter profiles from $600 \mathrm{MHz} \mathrm{NMR}$ data (black line) ${ }^{34}$ and iRED analysis of the 20 ns AMBER99 trajectory (green diamonds) and AMBER99SB (red triangles) trajectories. 

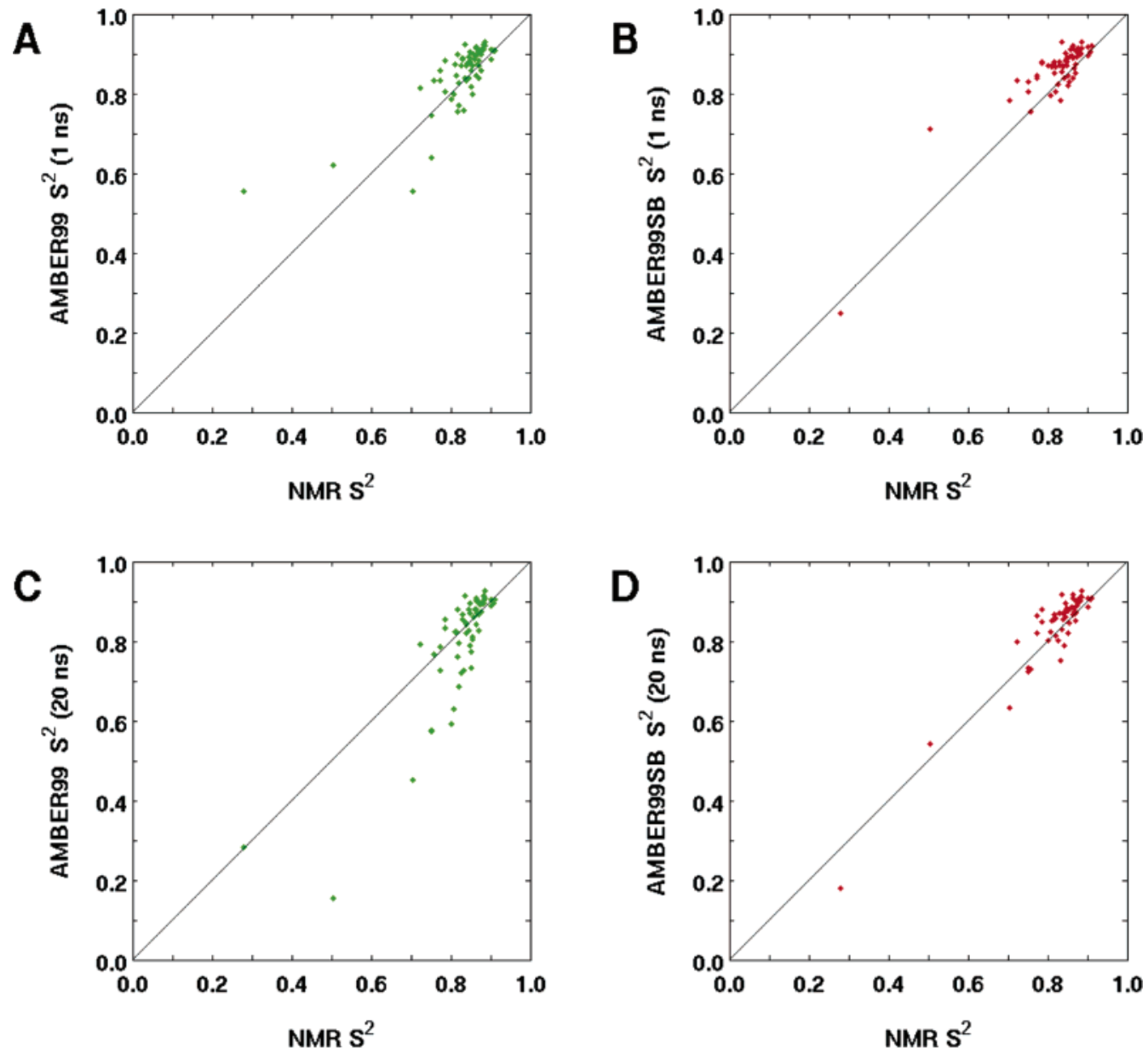

Figure 3. Ubiquitin backbone $\mathrm{N}-\mathrm{H}$ bond order parameter correlation plots. Order parameters calculated from the AMBER99 trajectory at $1 \mathrm{~ns}(\mathrm{~A})$, and $20 \mathrm{~ns}(\mathrm{C})$ and the AMBER99SB trajectory at $1 \mathrm{~ns}(\mathrm{~B})$ and $20 \mathrm{~ns}(\mathrm{D})$ are correlated with the same experimentally derived data set in all panels. ${ }^{34}$

problem lies with the force field itself rather than with poor sampling statistics, as is further demonstrated through the analysis of $10 \mathrm{~ns}$ subtrajectories of the AMBER99 simulation (see the Supporting Information). By contrast, it is clear from Figures 2 and 3D that the 20 ns AMBER99SB trajectory reproduces the dynamics of the mobile regions of ubiquitin well. This conclusion is supported by the decreasing RMSD and the approach of $\left\langle S_{\mathrm{MD}^{2}}{ }^{2} S_{\mathrm{NMR}^{2}}{ }^{2}>\right.$ to 1.0 (Table 2 ), by the similarity in the statistics from each of the four $5 \mathrm{~ns}$ subsets of the trajectory, and by more detailed analysis of $10 \mathrm{~ns}$ subtrajectories provided as Supporting Information.

This result is largely consistent with previously reported AMBER99SB ubiquitin and hen lysozyme simulations ${ }^{25}$ and a hen lysozyme trajectory computed with the CHARMM C22/CMAP force field. ${ }^{23}$ Both AMBER99SB and CHARMM C22/CMAP were produced from their parent force fields by adjusting the backbone $\varphi$ and $\psi$ torsion parameters, and the improvement at reproducing NMR order parameters indicates that these changes were made in the right direction. However, the question of whether these modified potentials properly sample the amplitudes and time scales of $\varphi$ and $\psi$ fluctuations on even longer time scales (tens of nanoseconds to milliseconds) cannot be addressed by comparison with spinrelaxation-derived $S^{2}$ values, which are insensitive to motions with correlation times slower than global tumbling. They will have to be validated using experimental data that are sensitive to these motions, such as residual dipolar couplings. ${ }^{53-57}$

3.3. Time-Correlation Functions and Relaxation Parameters. Time scales of internal dynamics can be extracted from reorientational correlation functions of each $\mathrm{N}-\mathrm{H}$ bond vector calculated from a trajectory according to eq 2 . For a well-sampled Markov process, the decay of the timecorrelation function to a plateau is (multi)exponential. For a finite trajectory, only if a correlation function is wellconverged does it approach a plateau value, which corresponds to the computed $S^{2}$ order parameter. Because the statistical error of a correlation function becomes often unacceptably large for times $t>T_{\mathrm{MD}} / 3$ (where $T_{\mathrm{MD}}$ is the total length of the trajectory), internal correlation functions $C_{\mathrm{I}}(t)$ are computed here up to times $0.3 T_{\mathrm{MD}}$. Even then, there is a finite chance for the occurrence of rare events, for example, a local torsion angle transition, which can lead to the absence of a plateau in $C_{\mathrm{I}}(t)$.

For the calculation of NMR relaxation data from a trajectory, high-quality correlation functions are required only for times $t \leq \tau_{\mathrm{c}}$. Therefore, the presence of rare events is inconsequential for the NMR relaxation parameter calculation 

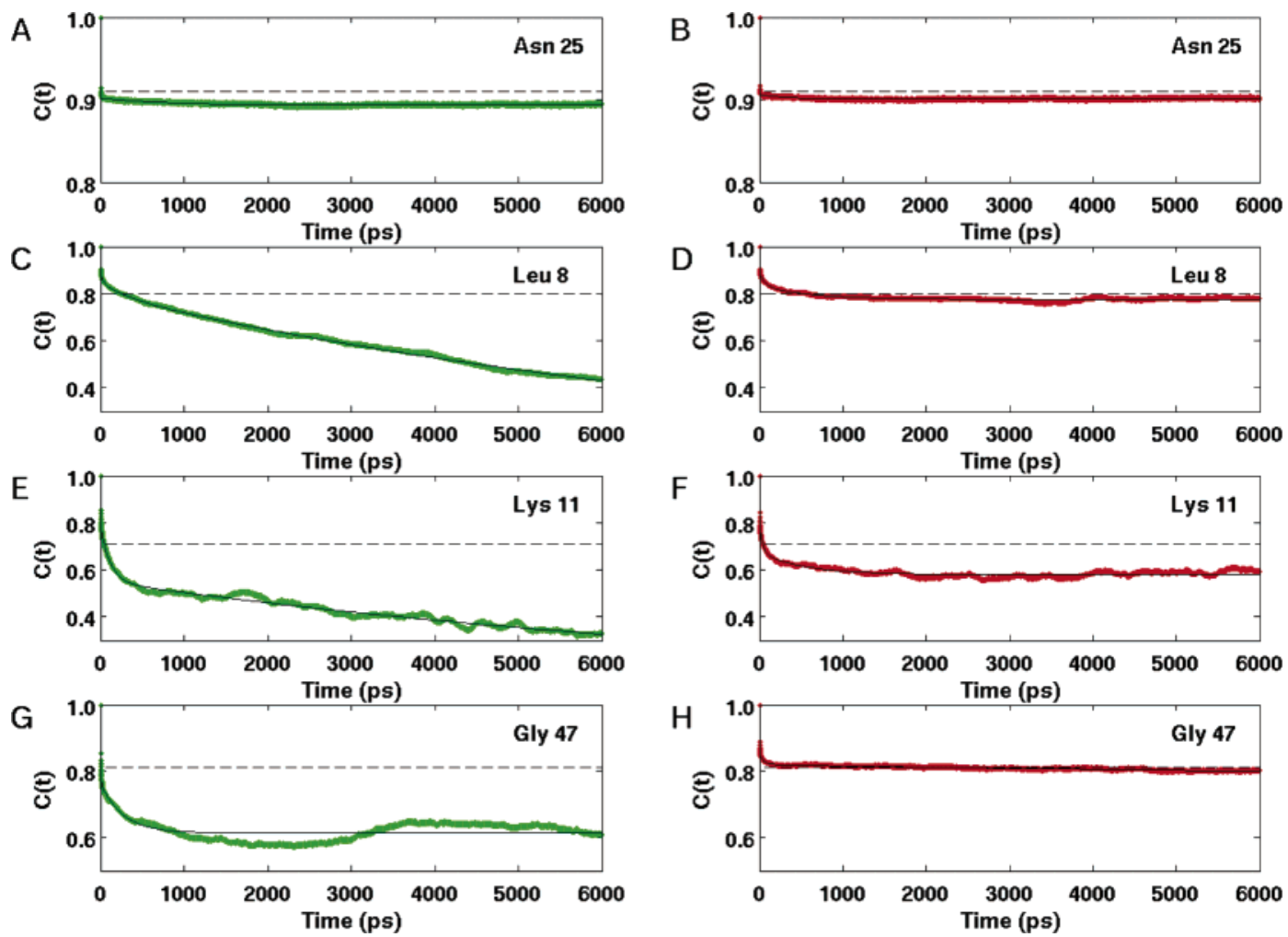

Figure 4. Ubiquitin backbone $\mathrm{N}-\mathrm{H}$ bond correlation functions from the $20 \mathrm{~ns} A M B E R 99$ trajectory $(A, C$, E, and $\mathrm{G})$ and AMBER99SB trajectory (B, D, F, and H). The green line (AMBER99) and the red line (AMBER99SB) are the correlation functions computed from the trajectories, and the thin lines are the best fit to the correlation functions generated using eq 9 . Best fit parameters for all correlation functions in this figure are presented in Table 3 (AMBER99) and Table 4 (AMBER99SB). The experimental order parameter ${ }^{34}$ is represented as a dashed line in each panel for reference.

as long as the average recurrence time of such events significantly exceeds $\tau_{\mathrm{c}}$ (4.1 ns for ubiquitin).

A set of 72 correlation functions $C_{\mathrm{I}}(t)$ has been computed for $t \leq 6 \mathrm{~ns}$, corresponding to each of the 72 backbone ${ }^{15} \mathrm{~N}-$ ${ }^{1} \mathrm{H}$ pairs for both $20 \mathrm{~ns}$ trajectories using AMBER99 and AMBER99SB. The correlation functions show different behaviors that can be subdivided into three distinct categories. The first category is exemplified by the correlation functions of Asn 25 (Figure 4A,B). They are characterized by an extremely rapid drop from $C_{\mathrm{I}}(0)=1$ to a value typically near 0.9 , followed by a rapid convergence to a stable plateau value corresponding to $S^{2}$. The very fast time-scale motions producing the initial decay reflect local librations. Their effect on spin relaxation rates is simply scaling by $S^{2}$; that is, the precise correlation times of these motions do not enter. Consequently, multiexponential fitting of these correlation functions to eq 9 yields only a few significantly nonzero $\tau_{k}$ and $A_{k}$ values, with $A_{0}$ closely matching $S^{2}$, as can be seen for Asn 25 in both the AMBER99 trajectory (Table 3) and the AMBER99SB trajectory (Table 4). Relaxation parameters of $\mathrm{N}-\mathrm{H}$ bond vectors that exhibit this type of correlation function are rather well-reproduced by both the AMBER99 and the AMBER99SB trajectories.

The second category of correlation functions exhibits a rapid drop at the beginning, similar to that found for the first category, followed by decay with a correlations time $\gg 1$ ps to a plateau $S^{2} \leq 0.85$. Examples of such correlation functions are those of Leu 8 (Figure 4D), Lys 11 (Figure 4F), and Gly 47 (Figure 4H) from the AMBER99SB simulation.

The third category of correlation functions is characterized by substantial deviation from multiexponential decay behavior without a clear indication of convergence toward a plateau. Excluding the C-terminal tail, 15 correlation functions computed from the AMBER99 trajectory fall into this category (Leu 8, Thr 9, Gly 10, Lys 11, Ser 20, Ile 36, Gln 41, Ala 46, Gly 47, Lys 48, Gln 49, Leu 50, Gly 53, Arg 54, and Thr 55). All of these residues but Ile 36 display large errors in their order parameters (Figure 2). The only core domain residues in the AMBER99SB trajectory that fall into this category are Ser 20, which is also one of the residues with the worst predicted order parameter from this trajectory, and Gln 41, which also has a predicted order parameter below the experimental value.

The two categories of correlation functions reflecting significant internal mobility (categories 2 and 3) can be illustrated for residues 7-11, which form the loop region of the $\mathrm{N}$-terminal $\beta$-hairpin. This is the most mobile region of ubiquitin, except for the C-terminal tail. Tables 3 and 4 include a summary of the fitting parameters for these residues from the AMBER99 and AMBER99SB trajectories, respectively. For all sites in this loop, $A_{0}$ is much lower than $S^{2}$ in the AMBER99 simulation, consistent with the absence of a plateau in the calculated correlation functions. Exponents with $\tau_{k}>1.0 \mathrm{~ns}$ are observed in these sites, contributing 
Table 3. Exponential Fitting Parameters for the 20 ns AMBER99 Trajectory

\begin{tabular}{|c|c|c|c|c|c|c|c|}
\hline $\begin{array}{l}\text { residue } \\
\text { number }\end{array}$ & $A_{0}$ & $\begin{array}{l}A_{1}{ }^{a} \\
\tau_{1}{ }^{b}\end{array}$ & $\begin{array}{l}A_{2} \\
\tau_{2}\end{array}$ & $\begin{array}{l}A_{3} \\
\tau_{3} \\
\end{array}$ & $\begin{array}{l}A_{4} \\
\tau_{4}\end{array}$ & $\begin{array}{l}A_{5} \\
\tau_{5}\end{array}$ & $S^{2}(\mathrm{NMR})^{c}$ \\
\hline \multirow[t]{2}{*}{7} & 0.733 & 0.103 & 0.025 & 0.006 & 0.027 & 0.107 & 0.850 \\
\hline & & 11387.104 & 192.394 & 192.392 & 12.236 & 0.013 & \\
\hline \multirow[t]{2}{*}{8} & 0.000 & 0.792 & 0.055 & 0.034 & 0.027 & 0.092 & 0.800 \\
\hline & & 9846.996 & 363.549 & 41.353 & 5.185 & 0.031 & \\
\hline \multirow[t]{2}{*}{9} & 0.364 & 0.360 & 0.000 & 0.089 & 0.060 & 0.128 & 0.750 \\
\hline & & 4201.172 & 1196.254 & 105.116 & 7.747 & 0.005 & \\
\hline \multirow[t]{2}{*}{10} & 0.460 & 0.195 & 0.000 & 0.000 & 0.125 & 0.219 & 0.750 \\
\hline & & 4109.661 & 3159.690 & 2904.702 & 126.644 & 0.015 & \\
\hline \multirow[t]{2}{*}{11} & 0.078 & 0.474 & 0.235 & 0.085 & 0.086 & 0.042 & 0.710 \\
\hline & & 9323.300 & 114.892 & 4.706 & 0.032 & 0.000 & \\
\hline \multirow[t]{2}{*}{25} & 0.894 & 0.009 & 0.073 & 0.008 & 0.009 & 0.008 & 0.910 \\
\hline & & 719.858 & 0.604 & 0.034 & 0.026 & 0.008 & \\
\hline \multirow[t]{2}{*}{47} & 0.614 & 0.149 & 0.117 & 0.016 & 0.087 & 0.017 & 0.810 \\
\hline & & 275.002 & 5.592 & 0.037 & 0.005 & 0.000 & \\
\hline
\end{tabular}

${ }^{a} A_{k}$ are the amplitudes of the corresponding exponential contributions to the correlation function (eq 9 ). ${ }^{b}$ Correlation times, $\tau_{k}$, are given in units of picoseconds. ${ }^{c}$ Lienin et al. ${ }^{34}$

Table 4. Exponential Fitting Parameters for the 20 ns AMBER99SB Trajectory

\begin{tabular}{|c|c|c|c|c|c|c|c|}
\hline $\begin{array}{l}\text { residue } \\
\text { number }\end{array}$ & $A_{0}$ & $\begin{array}{l}A_{1}^{a} \\
\tau_{1}{ }^{b}\end{array}$ & $\begin{array}{l}A_{2} \\
\tau_{2}\end{array}$ & $\begin{array}{l}A_{3} \\
\tau_{3}\end{array}$ & $\begin{array}{l}A_{4} \\
\tau_{4}\end{array}$ & $\begin{array}{l}A_{5} \\
\tau_{5}\end{array}$ & $S^{2}(\mathrm{NMR})^{c}$ \\
\hline \multirow[t]{2}{*}{7} & 0.854 & 0.005 & 0.024 & 0.004 & 0.022 & 0.091 & 0.850 \\
\hline & & 340.465 & 340.446 & 340.446 & 21.858 & 0.019 & \\
\hline \multirow[t]{2}{*}{8} & 0.775 & 0.008 & 0.006 & 0.048 & 0.056 & 0.108 & 0.800 \\
\hline & & 589.888 & 587.012 & 586.469 & 59.368 & 0.014 & \\
\hline \multirow[t]{2}{*}{9} & 0.693 & 0.002 & 0.006 & 0.077 & 0.149 & 0.000 & 0.750 \\
\hline & & 879.298 & 879.071 & 39.568 & 0.002 & 0.000 & \\
\hline \multirow[t]{2}{*}{10} & 0.693 & 0.005 & 0.004 & 0.037 & 0.084 & 0.176 & 0.750 \\
\hline & & 470.807 & 470.333 & 469.508 & 22.166 & 0.007 & \\
\hline \multirow[t]{2}{*}{11} & 0.580 & 0.003 & 0.090 & 0.001 & 0.131 & 0.195 & 0.710 \\
\hline & & 540.833 & 539.761 & 539.701 & 36.031 & 0.028 & \\
\hline \multirow[t]{2}{*}{25} & 0.901 & 0.006 & 0.068 & 0.008 & 0.009 & 0.008 & 0.910 \\
\hline & & 269.870 & 0.603 & 0.034 & 0.014 & 0.002 & \\
\hline \multirow[t]{2}{*}{47} & 0.778 & 0.042 & 0.033 & 0.042 & 0.080 & 0.024 & 0.810 \\
\hline & & 9355.259 & 49.153 & 4.560 & 0.009 & 0.009 & \\
\hline
\end{tabular}

${ }^{a} A_{k}$ are the amplitudes of the corresponding exponential contributions to the correlation function (eq 9). ${ }^{b}$ Correlation times, $\tau_{k}$, are given in units of picoseconds. ${ }^{c}$ Lienin et al. ${ }^{34}$

$20 \%$ or more of the total amplitude of $C_{\mathrm{I}}(t)$. In the examples of Leu 8 and Lys 11, a plateau is never actually reached due to the large amplitude contribution from low nanosecond time scale motion (Figure 4C, Table 3). While these motions have a notable effect on the calculated $S^{2}$ values, they are too slow to affect the relaxation parameters in a significant way. Therefore, the poor agreement between the spin relaxation parameters calculated from the AMBER99 trajectory and the experimental values (Figure 5) does not stem from these slower motions but, rather, from the largeamplitude motions on the subnanosecond time scale (note the presence of 100-400 ps $\tau_{k}$ associated with $A_{k}>0.1$ for these residues in Table 3 ).

The behavior of the correlation functions for the $\beta$-hairpin vectors is quite different in the AMBER99SB trajectory, as is summarized in Table 4. Although the values of $A_{0}$ are still noticeably lower than the experimental order parameters for most sites, they are all much higher than those of the AMBER99 trajectory. Similar to the AMBER99 trajectory, the order parameter calculated for Lys 11 deviates significantly from its experimental value, despite the fact that its correlation function is well-converged aside from being slightly noisier than some of the other correlation functions (Figure 4F). In addition to the influence of the underestimated order parameter, the discrepancy between back-calculated and experimental relaxation parameters for Lys 11 stems largely from the presence of a sizable 540 ps motional mode (Table 4), which is highly relaxation-active. Increasing the three $\tau_{k}$ of 540 ps to 2 ns without adjustment of any of the amplitudes $A_{k}$ results in a reduction of the back-calculated $T_{1}$ from 618 to $582 \mathrm{~ms}$ and $T_{2}$ from 239 to $230 \mathrm{~ms}$ and increases NOE from 0.511 to 0.647 . The agreement with the experimental values $\left(T_{1}=518 \mathrm{~ms}, T_{2}=205 \mathrm{~ms}\right.$, and $\mathrm{NOE}=0.608)$ is improved by this parameter adjustment, suggesting that the 540 ps motion dominating the backcalculated relaxation parameters in the simulation is absent in the experiment.

There are several sites outside of the $\beta$-hairpin which display significantly enhanced dynamics in the AMBER99 trajectory. The locations in primary sequence of residues with low order parameters in the AMBER99 trajectory and in the NB trajectory are similar but not identical (compare Figure 

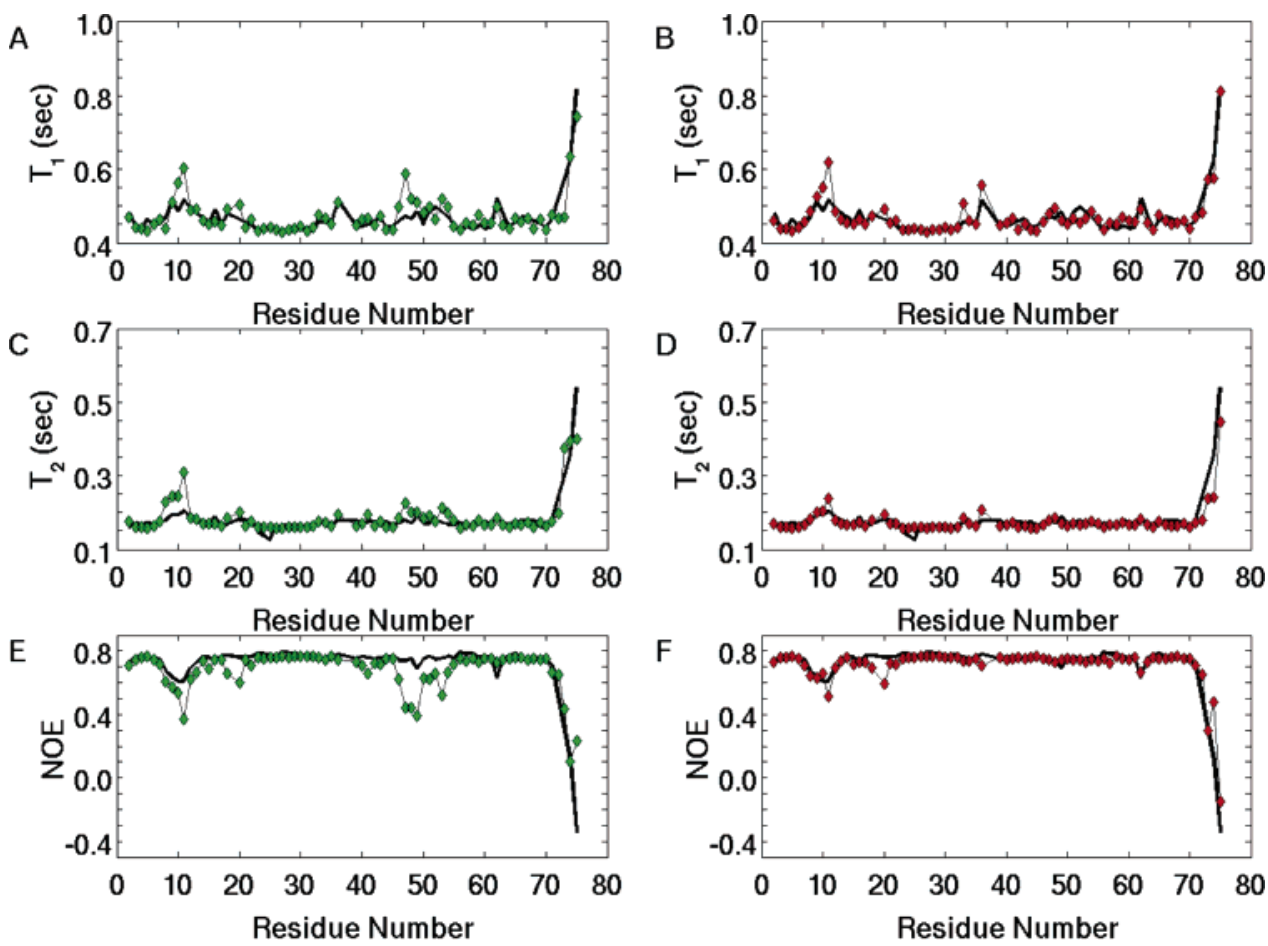

Figure 5. $600 \mathrm{MHz} \mathrm{T}_{1}, \mathrm{~T}_{2}$, and $\mathrm{NOE}$ data predicted from the $20 \mathrm{~ns}$ AMBER99 (A, C, and E) and AMBER99SB (B, D, and F) trajectories. Computed values are displayed as green (AMBER99) or red (AMBER99SB) diamonds along with the experimental values $^{34}$ as a black line for reference.

2 with Figure 6 of ref 28), whereas the discrepancy between the MD and experimental order parameters of these sites is reduced or eliminated for all sites in the AMBER99SB trajectory. For example, the correlation function for Gly 41 (Figure 4G,H) and its fit parameters (Tables 3 and 4) indicate a significant change in dynamics caused by the change in force field. Gly 47 has a relatively high experimental order parameter $\left(S^{2}=0.81\right),{ }^{34}$ which is very different from the value predicted from the AMBER99 simulation $\left(S^{2}=0.63\right)$. In contrast, the correlation function from the AMBER99SB trajectory for Gly 47 rapidly converges to a stable asymptote (Figure $4 \mathrm{H}$ ) and has a predicted order parameter of 0.82 , in excellent quantitative agreement with experimental results $\left(S^{2}=0.81\right){ }^{34}$

Calculating ${ }^{15} \mathrm{~N} T_{1}$ and ${ }^{15} \mathrm{~N} T_{2}$ spin relaxation times and heteronuclear NOEs is a stringent test of an MD trajectory's quality, because it requires that both the amplitude and the time scales of spin-relaxation active motion be reproduced well. For this purpose, the fit parameters from the correlation functions are used to generate spectral densities $J(\omega)$ for each $\mathrm{N}-\mathrm{H}$ bond vector along the ubiquitin backbone using eq 10. These spectral density functions are then used to calculate $T_{1}, T_{2}$, and NOE (eqs 11-13) for a magnetic field strength of $14.1 \mathrm{~T}$, which corresponds to the field strength at which the reference data set was measured. ${ }^{34}$ The results are shown in Figure 5. Note that residues 23-25 display chemical exchange contributions to $T_{2}$ which occur on the microsecond to millisecond time scale and are not modeled here.

On average, the AMBER99SB trajectory significantly better predicts $T_{1}, T_{2}$, and NOE than the AMBER99 trajectory (Table 5). At the level of individual residues, both the AMBER99 trajectory (Figure 5, left panels) and the AMBER99SB trajectory (Figure 5, right panels) show good agreement with experimental results for most of the rigid residues found in secondary structural elements. However, it is again apparent that the dynamics of the $\beta$-hairpin loop (residues 7-10) and numerous other loops are severely overestimated in the AMBER99 trajectory, consistent with the order parameter analysis. The NB trajectory was also used to back-calculate the relaxation parameters of ubiquitin, and the authors found poor agreement in similar regions. ${ }^{28}$ The AMBER99SB trajectory, on the other hand, reproduces the experimental relaxation times and heteronuclear NOEs well for most sites.

While the overall agreement between experimental and calculated values of $T_{1}, T_{2}$, and NOE is clearly higher for the AMBER99SB trajectory than for the AMBER99 trajectory, the different magnitudes of the individual parameters makes a quantitative comparison less straightforward. In analogy to the $Q$ value used for residual dipolar couplings, ${ }^{50}$ the parameters $Q_{T}$ and $Q_{R}$ presented in Table 5 (eq 14) allow direct quantitative comparison between the individual parameters, by renormalizing the RMSD to eliminate the influence of differences in parameter magnitudes. In general, $Q_{R}$ is lower than $Q_{T}$ because the influence of the C-terminal tail residues, which are less well-modeled even in the $20 \mathrm{~ns}$ AMBER99SB trajectory, is diminished in $Q_{R}$ as compared to $Q_{T}$. This effect is seen most clearly by comparing RMSD, $Q_{T}$, and $Q_{R}$ for the AMBER99SB trajectory.

The use of $Q_{T, R}$ demonstrates why comparison of simulation against experimental spin-relaxation times and heteronuclear Overhauser effects is a more objective measure of simulation quality than comparison with $S^{2}$ alone. Statistics and correlation plots (Table 2 and Figure 3 ) indicate that the 1 ns AMBER99 trajectory yields the highest-quality reproduction of the experimental order parameters of any 

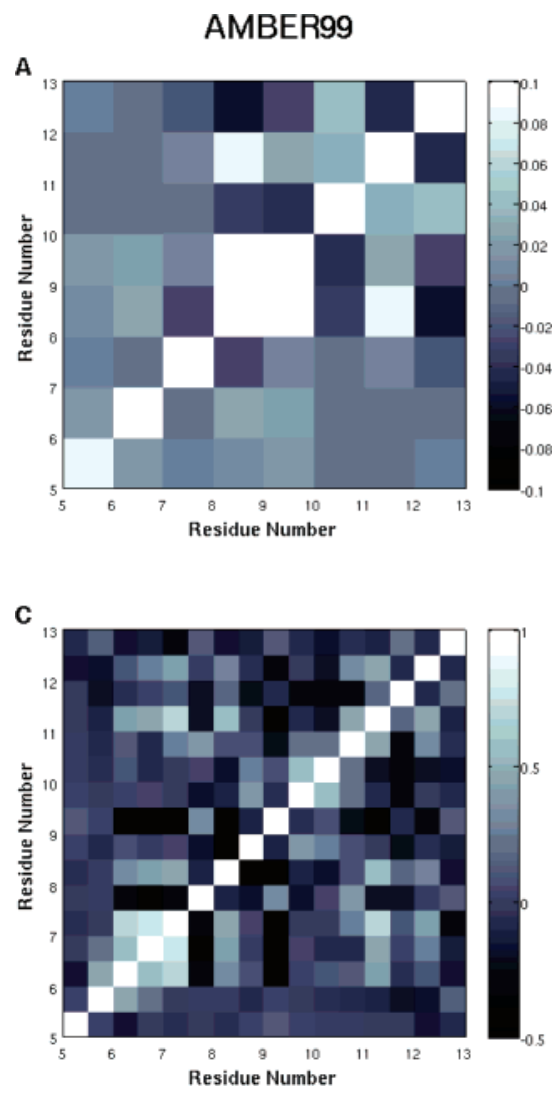
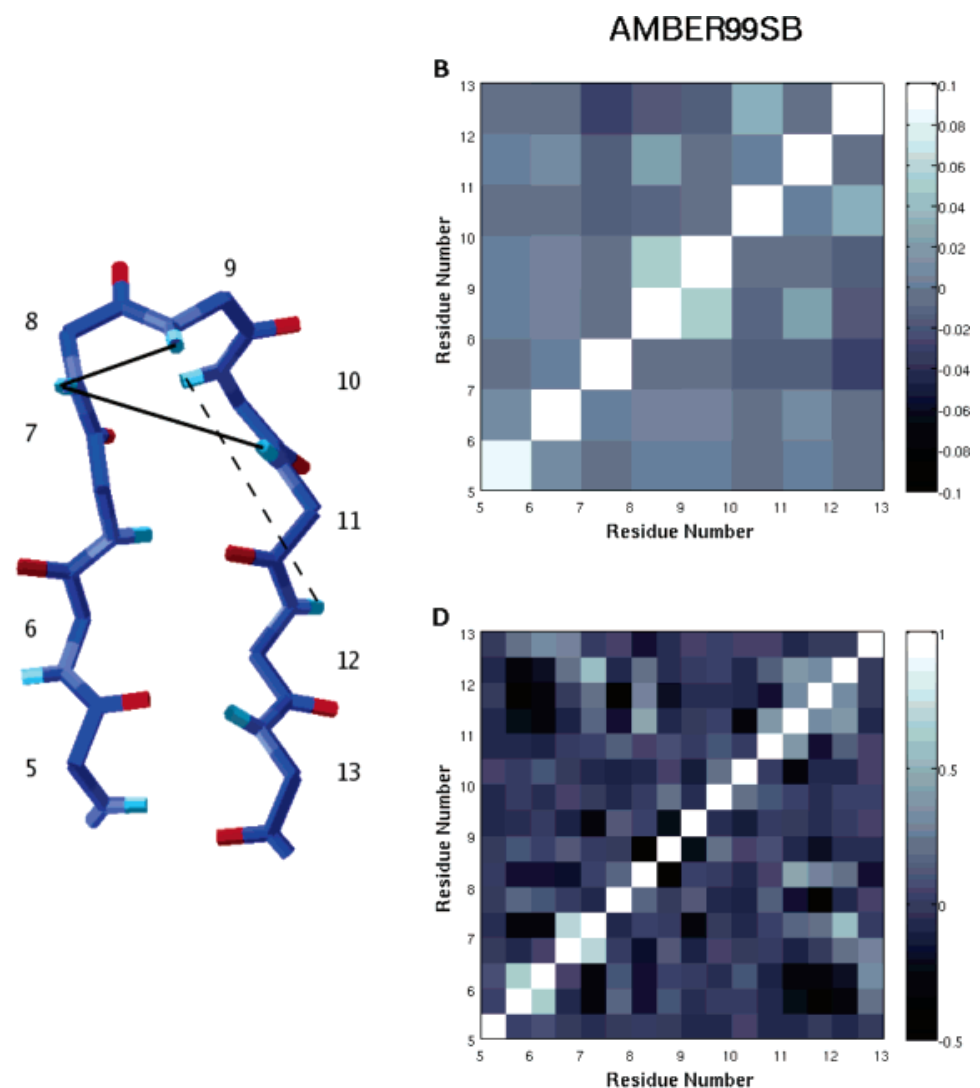

Figure 6. Internal motion correlations in the $\beta$-hairpin of ubiquitin (residues $5-12$ ) from the 20 ns AMBER99 and AMBER99SB trajectories. Rank 2 reorientational motion of backbone $\mathrm{N}-\mathrm{H}$ bond vectors is represented as the iRED covariance matrix for internal motion constructed only from the N-5 eigenvectors corresponding to internal motion for AMBER99 (A) and AMBER99SB (B). Correlations in the backbone $\varphi$ and $\psi$ torsion angles are represented by a cross-correlation matrix of the real part of eq 15 for AMBER99 (C) and AMBER99SB (D). The backbone structure diagram in the center of the figure displays the residues of the $\beta$-hairpin represented in panels $A-D$, with three key covariance interactions from the AMBER99SB iRED matrix mapped as solid lines (where the $\mathrm{N}-\mathrm{H}$ bond vectors project out of the page) and a dashed line (where the $\mathrm{N}-\mathrm{H}$ bond vectors project into the page). The imaginary part of the covariance matrix of eq 15 behaves similarly to the real part depicted in panels $C$ and $D$.

Table 5. Spin Relaxation Times and Heteronuclear Overhauser Effects Calculated from the $20 \mathrm{~ns}$ AMBER99 and AMBER99SB Trajectories with Respect to the Experimental Data

\begin{tabular}{lcccccc}
\hline \multicolumn{1}{c}{ data set ${ }^{a}$} & $R_{\mathrm{p}}{ }^{b}$ & $R_{\mathrm{S}}{ }^{c}$ & $\mathrm{RMSD}^{d}$ & $Q_{T^{e}}$ & $\left(Q_{R}\right)^{e}$ & $<\mathrm{MD} / \mathrm{NMR}>f$ \\
\hline AMBER99, $T_{1}$ & 0.866 & 0.744 & 0.029 & 0.061 & $(0.051)$ & $1.016 \pm 0.057$ \\
AMBER99, $T_{2}$ & 0.846 & 0.600 & 0.029 & 0.150 & $(0.091)$ & $1.031 \pm 0.118$ \\
AMBER99, NOE & 0.747 & 0.659 & 0.119 & 0.173 & $\sum=0.384(0.315)$ & \\
AMBER99SB, $T_{1}$ & 0.913 & 0.729 & 0.024 & 0.050 & $(0.043)$ & $1.006 \pm 0.048$ \\
AMBER99SB, $T_{2}$ & 0.943 & 0.635 & 0.022 & 0.119 & $(0.062)$ & $0.981 \pm 0.072$ \\
AMBER99SB, NOE & 0.938 & 0.517 & 0.066 & 0.091 & $1.030 \pm 0.153$ \\
\end{tabular}

${ }^{a}$ Residues lle 21 and Asn 25 are excluded from the calculation because of an exchange contribution to the experimental $T_{2}$ which would not be reproduced in the simulation. ${ }^{b}$ Pearson's correlation coefficient between the simulation and the experimental set. ${ }^{34}{ }^{c}$ Spearman's rank correlation coefficient between the simulation and the experimental set. ${ }^{34}{ }^{d}$ Root-mean-square deviation between the simulation and the experimental set. ${ }^{34}$ e $Q$ value between the simulation and the experimental set calculated from eq 14 . Values in parenthesis are calculated from $R_{1}, R_{2}$, and NOE. ${ }^{f}$ Average is taken over all residues for which there is both MD and experimental data.

subtrajectory from that simulation, and yet the total $Q_{R}$ for that trajectory $\left(Q_{R}=0.453\right)$ is clearly worse than that for the full $20 \mathrm{~ns}$ trajectory $\left(Q_{R}=0.315\right)$. This demonstrates that an order parameter comparison, while informative, does not provide a quantitative assessment of the quality of a trajectory because it ignores the potentially complex interplay between time scale and amplitude effects.
3.4. Dynamics of the $\beta$-Hairpin Loop. Having demonstrated that the AMBER99SB simulation reproduces well experimental order parameters, spin relaxation times, and heteronuclear Overhauser effects, the trajectory is used to study the dynamics of the turn of the $\beta$-hairpin near the $\mathrm{N}$-terminus of ubiquitin in more detail. Application of the RED protocol, which is a variant of the iRED protocol used 


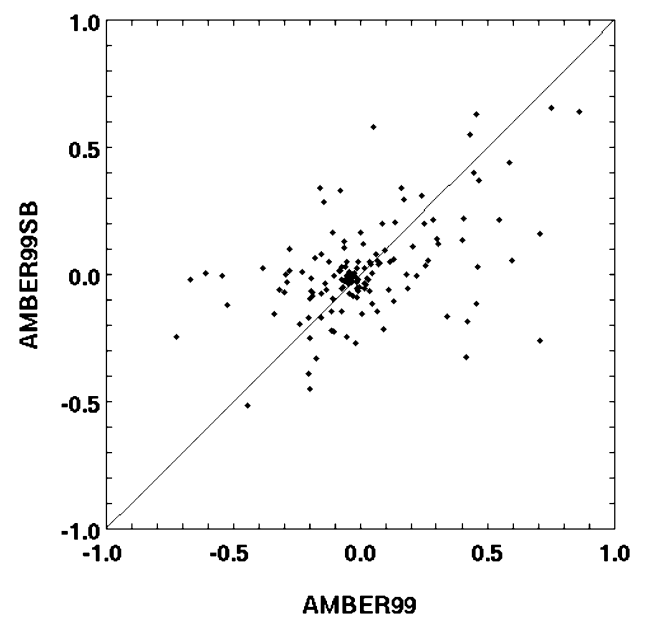

Figure 7. Comparison of torsion-angle correlation coefficients between $\beta$-hairpin matrices using AMBER99 (Figure 6C) and AMBER99SB (Figure 6D). The average correlation coefficients are $0.017 \pm 0.270$ for AMBER99 and $0.013 \pm 0.196$ for AMBER99SB. The linear correlation coefficient between the AMBER99 and the AMBER99SB correlations is $R_{\mathrm{p}}=0.48$.

in the present study, did previously show correlations in the reorientational motions of various residues in the $\beta$-hairpin loop including correlations between many of the backbone torsional degrees of freedom. ${ }^{37}$ Optimization of a combined reorientational and torsional covariance matrix against experimental spin relaxation data resulted in a clear reduction of the amount of correlations among the torsional degrees of freedom. ${ }^{37}$

Similarly to the previous study, both the portion of the iRED covariance matrix corresponding to internal reorientations of the $\beta$-hairpin and a matrix of correlation coefficients between the $\varphi$ and $\psi$ torsion angles for the same region constructed from the AMBER99 trajectory show significant (negative) correlations spanning the loop and its joining $\beta$ strands (Figure 6A,C). In contrast, the same matrices computed from the AMBER99SB trajectory suggest lower correlations between these sites (Figure 6B,D). The matrices in Figure $6 \mathrm{~A}$ and $\mathrm{B}$ are constructed by removing the eigenmodes corresponding to global reorientation from the total iRED covariance matrix, and their diagonal elements are proportional to $1-S^{2}$ (eq 8 ). Figures 6 and 7 show that motional correlations are differentially altered and reduced on average in the AMBER99SB trajectory relative to the AMBER99 trajectory. Because it is difficult to experimentally quantify these motional correlations (off diagonal elements of the matrices in Figure 6), validation of the simulated correlations is not feasible at this time. Altered correlations are likely to have ramifications for estimating thermodynamic quantities, in particular, configurational entropies, from trajectories using AMBER99SB.

\section{Conclusions}

Comparison of MD simulations against NMR spin-relaxation data represents a rigorous quality test of MD simulation protocols and force fields. Presently available computational power enables routine calculation of molecular dynamics trajectories of biomolecules extending into the tens of nanoseconds range. These simulations are reaching the point where they allow one to systematically assess and distinguish between finite conformational sampling effects and the accuracy of the force field itself.

Comparisons with NMR order parameters have long suggested that the amount of dynamics of protein loops are systematically overestimated in MD trajectories. Recent modifications of backbone potentials of both the CHARMM C22 force field and the AMBER99 force field produce significant improvements for both force fields. ${ }^{22-25}$ Due to the distinct sensitivity of the experimental NMR $S^{2}$ order parameters to the associated motional time scales, their usefulness as a comparison metric between simulation and relaxation data is somewhat limited. Many computed $S^{2}$ values depend on the duration of the trajectory with longer trajectories displaying on average lower computed order parameters. This is consistent with experimental evidence from residual dipolar couplings measured in multiple alignment media suggesting the presence of additional motions on nansecond to millisecond time scales that are not captured by $T_{1}$-type relaxation data. ${ }^{53,55-57}$ At present, such motions cannot be comprehensively probed by standard MD simulations; therefore, they will require other methods such as enhanced sampling techniques.

We demonstrate here that comparison directly against the "raw" relaxation parameters $T_{1}, T_{2}$, and NOE is complementary to and for fully quantitative purposes preferable over the use of $S^{2}$ parameters to assess the performance of MD simulations. Comparison based on relaxation parameters implicitly includes the complex time-scale effects of the motions on spin-relaxation parameters. While internal motional correlation times can be separately extracted from a model-free analysis ${ }^{17,18}$ or alternative treatments, ${ }^{58}$ they are typically much less well-defined than the corresponding order parameters, and they also depend on the model selection procedure. $^{59}$

Because overall rotational tumbling is neither properly reproduced by current force fields nor properly sampled by trajectories in the tens of nanoseconds, a hybrid approach is used here that combines the experimentally derived overall tumbling correlation function $C_{\mathrm{O}}(t)$ with the internal correlation function $C_{\mathrm{I}}(t)$ calculated from the trajectory. A drawback of the method is that the relaxation parameters cannot be meaningfully predicted without accurate knowledge of the overall tumbling correlation time. However, if fit parameters using eq 9 to the internal correlation functions of MD trajectories were systematically reported in future simulations, such as the ones of Tables 3 and 4, relaxation parameters can be readily back-calculated once experimental relaxation data are available, either by using the experimentally determined overall tumbling correlation time, $\tau_{\mathrm{c}}$, or by treating $\tau_{\mathrm{c}}$ as a fit parameter that optimizes agreement between the experiment and simulation. Such a procedure is a special case to the reporting of the dominant part of iRED eigenmodes together with their corresponding correlation times, from which the overall tumbling time can be modeled by fitting the correlation times of the five eigenmodes with the largest eigenvalues to the experimental data. ${ }^{38}$ While in the present treatment isotropic overall tumbling is 
assumed, which is adequate for ubiquitin, ${ }^{33}$ it can be easily extended to axially symmetric or fully anisotropic overall tumbling motion. ${ }^{60}$

We find that for ubiquitin the modified AMBER99SB force field is better at reproducing both the amplitude and time scale of spin-relaxation active internal motions than AMBER99. The overall reproduction of NMR relaxation times and the heteronuclear NOE, as well as NMR order parameters, using this force field is good, which includes the loop region of the $\mathrm{N}$-terminal $\beta$-hairpin. The reduction in motional amplitudes is accompanied by both a slowdown of the intramolecular dynamics and a reduction of torsion angle correlations. Some discrepancies still remain with AMBER99SB, indicating that there is room for further improvement. Moreover, the rate of macromolecular global tumbling is systematically overestimated in MD simulations using most current water models, which will require improvements of the way explicit solvent water is modeled and how water molecules interact with the solute. Such advances would be particularly beneficial for assessing simulations of (partially) unfolded and disordered protein systems whose overall motion is not separable from internal dynamics and for which an internal order parameter is not defined any longer. ${ }^{38,61,62}$

Acknowledgment. S.A.S. is the recipient of an NIH postdoctoral fellowship. This work was supported by the NSF (grant 0621482).

Supporting Information Available: The convergence of the 20 ns AMBER99 and AMBER99SB trajectories validated by detailed comparison of dynamics information generated from the first and the second half of each trajectory. This material is available free of charge via the Internet at http://pubs.acs.org.

\section{References}

(1) Case, D. A. Molecular Dynamics and NMR Spin Relaxation in Proteins. Acc. Chem. Res. 2002, 35, 325-331.

(2) Brüschweiler, R. New Approaches to the Dynamic Interpretation and Prediction of NMR Relaxation Data from Proteins. Curr. Opin. Struct. Biol. 2003, 13, 175-183.

(3) Palmer, A. G. NMR Characterization of the Dynamics of Biomacromolecules. Chem. Rev. 2004, 104, 3623-3640.

(4) Jarymowycz, V. A.; Stone, M. J. Fast Time Scale Dynamics of Protein Backbones: NMR Relaxation Methods, Applications, and Functional Consequences. Chem. Rev. 2006, 106, 1624-1671.

(5) Adcock, S. A.; McCammon, J. A. Molecular Dynamics: Survey of Methods for Simulating the Activity of Proteins. Chem. Rev. 2006, 106, 1589-1615.

(6) Chatfield, D. C.; Szabo, A.; Brooks, B. R. Molecular Dynamics of Staphylococcal Nuclease: Comparison of Simulation with N-15 and C-13 NMR Relaxation Data. $J$. Am. Chem. Soc. 1998, 120, 5301-5311.

(7) Chandrasekhar, I.; Clore, G. M.; Szabo, A.; Gronenborn, A. M.; Brooks, B. R., A 500-ps Molecular Dynamics Simulation Study of Interleukin- $1 \beta$ in Water: Correlation with Nuclear Magnetic Resonance Spectroscopy and Crystallography. $J$. Mol. Biol. 1992, 226, 239-250.
(8) Palmer, A. G.; Case, D. A. Molecular Dynamics Analysis of NMR Relaxation in a Zinc Finger Peptide. J. Am. Chem. Soc. 1992, 114, 9059-9067.

(9) Wong, K. B.; Daggett, V. Barstar Has a Highly Dynamic Hydrophobic Core: Evidence from Molecular Dynamics Simulations and Nuclear Magnetic Resonance Relaxation Data. Biochemistry 1998, 37, 11182-11192.

(10) Stocker, U.; van Gunsteren, W. F. Molecular Dynamics Simulation of Hen Egg White Lysozyme: A Test of the GROMOS96 Force Field against Nuclear Magnetic Resonance Data. Proteins: Struct., Funct., Genet. 2000, 40, 145153.

(11) Prompers, J. J.; Scheurer, C.; Brüschweiler, R. Characterization of NMR Relaxation-Active Motions of a Partially Folded A-State Analogue of Ubiquitin. J. Mol. Biol. 2001, 305, 1085-1097.

(12) Showalter, S. A.; Hall, K. B. A Functional Role for Correlated Motion in the N-Terminal RNA-Binding Domain of Human U1A Protein. J. Mol. Biol. 2002, 322, 533-542.

(13) Pang, Y.; Buck, M.; Zuiderweg, E. R. P. Backbone Dynamics of the Ribonuclease Binase Active Site Area Using Multinuclear (N-15 and (CO)-C-13) NMR Relaxation and Computational Molecular Dynamics. Biochemistry 2002, 41, 2655-2666.

(14) Philippopoulos, M.; Mandel, A. M.; Palmer, A. G.; Lim, C. Accuracy and Precision of NMR Relaxation Experiments and MD Simulations for Characterizing Protein Dynamics. Proteins: Struct., Funct., Bioinf. 1997, 28, 481-493.

(15) Radkiewicz, J. L.; Brooks, C. L. Protein Dynamics in Enzymatic Catalysis: Exploration of Dihydrofolate Reductase. J. Am. Chem. Soc. 2000, 122, 225-231.

(16) Pfeiffer, S.; Fushman, D.; Cowburn, D. Simulated and NMRDerived Backbone Dynamics of a Protein with Significant Flexibility: A Comparison of Spectral Densities for the Beta ARK PH Domain. J. Am. Chem. Soc. 2001, 123, 30213036.

(17) Lipari, G.; Szabo, A. Model-Free Approach to the Interpretation of Nuclear Magnetic Resonance Relaxation in Macromolecules. 1. Theory and Range of Validity. J. Am. Chem. Soc. 1982, 104, 4546-4559.

(18) Lipari, G.; Szabo, A. Model-Free Approach to the Interpretation of Nuclear Magnetic Resonance Relaxation in Macromolecules. 2. Analysis of Experimental Results. J. Am. Chem. Soc. 1982, 104, 4559-4570.

(19) Zhang, F. L.; Brüschweiler, R. Contact Model for the Prediction of NMR N-H Order Parameters in Globular Proteins. J. Am. Chem. Soc. 2002, 124, 12654-12655.

(20) Ming, D. M.; Brüschweiler, R. Prediction of Methyl-Side Chain Dynamics in Proteins. J. Biomol. NMR 2004, 29, 363368.

(21) Abergel, D.; Bodenhausen, G. Predicting Internal Protein Dynamics from Structures Using Coupled Networks of Hindered Rotators. J. Chem. Phys. 2005, 123, 204901.

(22) Mackerell, A. D. Empirical Force Fields for Biological Macromolecules: Overview and Issues. J. Comput. Chem. 2004, 25, 1584-1604.

(23) Buck, M.; Bouguet-Bonnet, S.; Pastor, R. W.; MacKerell, A. D. Importance of the CMAP Correction to the CHARMM22 Protein Force Field: Dynamics of Hen Lysozyme. Biophys. J. 2006, 90, L36-L38. 
(24) Hornak, V.; Okur, A.; Rizzo, R. C.; Simmerling, C. HIV-1 Protease Flaps Spontaneously Open and Reclose in Molecular Dynamics Simulations. Proc. Natl. Acad. Sci. U.S.A. 2006, 103, 915-920.

(25) Hornak, V.; Abel, R.; Okur, A.; Strockbine, B.; Roitberg, A.; Simmerling, C. Comparison of Multiple AMBER Force Fields and Development of Improved Protein Backbone Parameters. Proteins: Struct., Funct., Bioinf. 2006, 65, 712725 .

(26) Brüschweiler, R.; Roux, B.; Blackledge, M.; Griesinger, C.; Karplus, M.; Ernst, R. R. Influence of Rapid Intramolecular Motion on NMR Cross-Relaxation Rates: A Molecular Dynamics Study of Antamanide in Solution. J. Am. Chem. Soc. 1992, 114, 2289-2302.

(27) Bremi, T.; Brüschweiler, R.; Ernst, R. R. A Protocol for the Interpretation of Side-Chain Dynamics Based on NMR Relaxation: Application to Phenylalanines in Antamanide. J. Am. Chem. Soc. 1997, 119, 4272-4284.

(28) Nederveen, A. J.; Bonvin, A. M. J. J. NMR Relaxation and Internal Dynamics of Ubiquitin from a $0.2 \mu$ s MD Simulation. J. Chem. Theory Comput. 2005, 1, 363-374.

(29) Peng, J. W.; Wagner, G. Mapping of Spectral Density Functions Using Heteronuclear NMR Relaxation Measurements. J. Magn. Reson. 1992, 98, 308-332.

(30) Case, D. A.; Cheatham, T. E.; Darden, T.; Gohlke, H.; Luo, R.; Merz, K. M.; Onufriev, A.; Simmerling, C.; Wang, B.; Woods, R. J. The AMBER Biomolecular Simulation Programs. J. Comput. Chem. 2005, 26, 1668-1688.

(31) Wang, J. M.; Cieplak, P.; Kollman, P. A. How Well Does a Restrained Electrostatic Potential (RESP) Model Perform in Calculating Conformational Energies of Organic and Biological Molecules? J. Comput. Chem. 2000, 21, 1049-1074.

(32) Schneider, D. M.; Dellwo, M. J.; Wand, A. J. Fast Internal Main Chain Dynamics of Human Ubiquitin. Biochemistry 1992, 31, 3645-3652.

(33) Tjandra, N.; Szabo, A.; Bax, A. Protein Backbone Dynamics and N-15 Chemical Shift Anisotropy from Quantitative Measurement of Relaxation Interference Effects. J. Am. Chem. Soc. 1996, 118, 6986-6991.

(34) Lienin, S. F.; Bremi, T.; Brutscher, B.; Brüschweiler, R.; Ernst, R. R. Anisotropic Intramolecular Backbone Dynamics of Ubiquitin Characterized by NMR Relaxation and MD Computer Simulation. J. Am. Chem. Soc. 1998, 120, 98709879.

(35) Chang, S. L.; Tjandra, N. Temperature Dependence of Protein Backbone Motion from Carbonyl C-13 and Amide N-15 NMR Relaxation. J. Magn. Reson. 2005, 174, 43-53.

(36) Ryabov, Y.; Fushman, D. Interdomain Mobility in Diubiquitin Revealed by NMR. Proteins: Struct., Funct., Bioinf. 2006, 63, 787-796.

(37) Prompers, J. J.; Brüschweiler, R. Reorientational Eigenmode Dynamics: A Combined MD/NMR Relaxation Analysis Method for Flexible Parts in Globular Proteins. J. Am. Chem. Soc. 2001, 123, 7305-7313.

(38) Prompers, J. J.; Brüschweiler, R. General Framework for Studying the Dynamics of Folded and Nonfolded Proteins by NMR Relaxation Spectroscopy and MD Simulation. $J$. Am. Chem. Soc. 2002, 124, 4522-4534.

(39) Daura, X.; Mark, A. E.; van Gunsteren, W. F. Parametrization of Aliphatic CHn United Atoms of GROMOS96 Force Field. J. Comput. Chem. 1998, 19, 535-547.
(40) Darden, T.; York, D.; Pedersen, L. Particle Mesh Ewald An $\mathrm{N} \log (\mathrm{N})$ Method for Ewald Sums in Large Systems. $J$. Chem. Phys. 1993, 98, 10089-10092.

(41) Vijaykumar, S.; Bugg, C. E.; Cook, W. J. Structure of Ubiquitin Refined at $1.8 \AA$ A Resolution. J. Mol. Biol. 1987, 194, 531-544.

(42) Jorgensen, W. L.; Chandrasekhar, J.; Madura, J. D.; Impey, R. W.; Klein, M. L. Comparison of Simple Potential Functions for Simulating Liquid Water. J. Chem. Phys. 1983, 79, 926-935.

(43) Ryckaert, J. P.; Ciccotti, G.; Berendsen, H. J. C. Numerical Integration of Cartesian Equations of Motion of a System with Constraints: Molecular Dynamics of n-Alkanes. $J$. Comput. Phys. 1977, 23, 327-341.

(44) Wangsness, R. K.; Bloch, F. The Dynamical Theory of Nuclear Induction. Phys. Rev. 1953, 89, 728-739.

(45) Bloch, F. Dynamical Theory of Nuclear Induction 2. Phys. Rev. 1956, 102, 104-135.

(46) Redfield, A. G. On the Theory of Relaxation Processes. IBM J. Res. Dev. 1957, 1, 19-31.

(47) Redfield, A. G. The Theory of Relaxation Processes. Adv. Magn. Reson. 1965, 1, 1-32.

(48) Brüschweiler, R.; Wright, P. E. NMR Order Parameters of Biomolecules - A New Analytical Representation and Application to the Gaussian Axial Fluctuation Model. J. Am. Chem. Soc. 1994, 116, 8426-8427.

(49) Abragam, A. Principles of Nuclear Magnetism; Clarendon Press: Oxford, U. K., 1961.

(50) Ottiger, M.; Bax, A. Bicelle-Based Liquid Crystals for NMRMeasurement of Dipolar Couplings at Acidic and Basic $\mathrm{pH}$ Values. J. Biomol. NMR 1999, 13, 187-191.

(51) Wang, J.; Brüschweiler, R. 2D Entropy of Discrete Molecular Ensembles. J. Chem. Theory Comput. 2006, 2, 18-24.

(52) Smith, L. J.; Mark, A. E.; Dobson, C. M.; van Gunsteren, W. F. Comparison of MD Simulations and NMR Experiments for Hen Lysozyme: Analysis of Local Fluctuations, Cooperative Motions, and Global Changes. Biochemistry 1995, 34, 10918-10931.

(53) Blackledge, M. Recent Progress in the Study of Biomolecular Structure and Dynamics in Solution from Residual Dipolar Couplings. Prog. Nucl. Magn. Reson. Spectrosc. 2005, 46, $23-61$.

(54) Bax, A.; Grishaev, A. Weak Alignment NMR: A HawkEyed View of Biomolecular Structure. Curr. Opin. Struct. Biol. 2005, 15, 563-570.

(55) Meiler, J.; Prompers, J. J.; Peti, W.; Griesinger, C.; Brüschweiler, R. Model-Free Approach to the Dynamic Interpretation of Residual Dipolar Couplings in Globular Proteins. J. Am. Chem. Soc. 2001, 123, 6098-6107.

(56) Bouvignies, G.; Bernado, P.; Meier, S.; Cho, K.; Grzesiek, S.; Brüschweiler, R.; Blackledge, M. Identification of Slow Correlated Motions in Proteins Using Residual Dipolar and Hydrogen-Bond Scalar Couplings. Proc. Natl. Acad. Sci. U.S.A. 2005, 102, 13885-13890.

(57) Tolman, J. R.; Ruan, K. NMR Residual Dipolar Couplings as Probes of Biomolecular Dynamics. Chem. Rev. 2006, 106, $1720-1736$. 
(58) Meirovitch, E.; Shapiro, Y. E.; Polimeno, A.; Freed, J. H. Protein Dynamics from NMR: The Slowly Relaxing Local Structure Analysis Compared with Model-Free Analysis. $J$. Phys. Chem. A 2006, 110, 8366-8396.

(59) Mandel, A. M.; Akke, M.; Palmer, A. G. Backbone Dynamics of Escherichia coli Ribonuclease H1: Correlations with Structure and Function in an Active Enzyme. J. Mol. Biol. 1995, 246, 144-163.

(60) Woessner, D. Nuclear Spin Relaxation in Ellipsoids Undergoing Rotational Brownian Motion. J. Chem. Phys. 1962, 37, 647-654.
(61) Brutscher, B.; Brüschweiler, R.; Ernst, R. R. Backbone Dynamics and Structural Characterization of the Partially Folded A State of Ubiquitin by H-1, C-13, and N-15 Nuclear Magnetic Resonance Spectroscopy. Biochemistry 1997, 36, 13043-13053.

(62) Dyson, H. J.; Wright, P. E. Unfolded Proteins and Protein Folding Studied by NMR. Chem. Rev. 2004, 104, 36073622.

CT7000045 